\title{
Inertial modes in stratified rotating neutron stars : An evolutionary description
}

\author{
L. Villain ${ }^{1,2,3}$, S. Bonazzola ${ }^{1}$ and P. Haensel ${ }^{2}$ \\ ${ }^{1}$ Laboratoire de l'Univers et de ses Théories (UMR 8102 du C.N.R.S.), Observatoire de Paris - \\ Section de Meudon, F-92195 Meudon Cedex, France \\ ${ }^{2}$ Copernicus Astronomical Center (CAMK), Polish Academy of Sciences, Bartycka 18, PL-00-716 Warszawa, Poland \\ ${ }^{3}$ Departament d'Astronomia i Astrofísica, Universitat de València, E-46100 Burjassot, Spain
}

(Dated: July 13, 2018)

\begin{abstract}
With (nonbarotropic) equations of state valid even when the neutron, proton and electron content of neutron star cores is not in beta equilibrium, we study inertial and composition gravity modes of relativistic rotating neutron stars. We solve the relativistic Euler equations in the time domain with a three dimensional numerical code based on spectral methods, in the slow rotation, relativistic Cowling and anelastic approximations. Principally, after a short description of the gravity modes due to smooth composition gradients, we focus our analysis on the question of how the inertial modes are affected by nonbarotropicity of the nuclear matter. In our study, the deviation with respect to barotropicity results from the frozen composition of nonsuperfluid matter composed of neutrons, protons and electrons, when beta equilibrium is broken by millisecond oscillations. We show that already for moderately fast rotating stars the increasing coupling between polar and axial modes makes those two cases less different than for very slowly rotating stars. In addition, as we directly solve the Euler equations, without coupling only a few number of spherical harmonics, we always find, for the models that we use, a discrete spectrum for the $l=m=2$ inertial mode. Finally, we conclude that, for nonbarotropic stars, the frequency of this mode, which is our main focus, decreases in a non-negligible way, whereas the time dependence of the energy transfer between polar and axial modes is substantially different due to the existence of low-frequencies gravity modes.
\end{abstract}

\section{INTRODUCTION}

Since the result of Andersson [1] and Friedman \& Morsink [2], numerous works have dealt with inertial modes of rotating relativistic stars (see reviews by Friedman \& Lockitch [3], Andersson [4] and Kokkotas \& Ruoff [5]). In perfect fluids, these modes have indeed been proven to satisfy the Chandrasekhar-Friedman-Schutz (CFS; [ 6] and [] ) criterion for instability whatever the angular velocity of the star is. Hence, due to their coupling to the gravitational field, these oscillations make neutron stars (NSs) very promising sources of gravitational waves $(\mathrm{GW})$. Yet, as NSs are complex relativistic objects, far from perfect fluids, the astrophysical relevance of the instability of inertial modes in rotating NSs is still an open issue. The final answer to this question will depend on the many physical phenomena that take place within NSs: their initial state at birth [8], existence of differential rotation [9], of a crust (10], 11], 12], 13]), of a huge magnetic field [14], of superfluid states of nucleons ([15], 16], 17]), of exotic particles, etc. Understanding better that potential instability is important for two main reasons. First, if it were proven to be relevant, it could have a great impact on our idea about the evolution of NSs and might explain the relatively slow angular velocity of observed pulsars. Moreover, in order for the data collected by GW detectors like VIRGO or LIGO to be useful, they have to be compared with predicted signals, such as the signal that results from the instability of inertial modes. Since theoretical predictions depend on the models used for the inner structure of NSs, this confrontation could possibly be as instructive for nuclear physics at high density as was helioseismology for neutrino physics.

In a previous article [18] (referred to here as Paper I), we have presented a spectral hydrodynamical code, which uses spherical coordinates, with the aim of studying time evolution of inertial modes in slowly rotating neutron stars. Having discussed the motivation for the slow rotation approximation in this previous article (it is still good even for the fastest known pulsars, see Paper I), we keep working with it in the present paper. Thus, the next step in our project was to improve the linear study of relativistic inertial modes by trying to take into account in a more realistic way the microphysical conditions that occur inside actual NSs. Among them, we retained the stratification of the star. It was quite easy to implement, and it is well-known that inertial modes change drastically depending on whether the equation of state (EOS) that describes the NS is barotropic or nonbarotropic ${ }^{1}$, while the status of purely axial modes of relativistic nonbarotropic NSs is still under debate $([\underline{3}],[\underline{5}])$. In addition, it is easy to show that, for the most basic model of a star built with the so-called "npe" matter, the assumption of a barotropic EOS, even if easier to use, is wrong as soon as the star is cold enough and the matter not in a stationary state. Hence, here we consider the time evolution of inertial modes in slowly

\footnotetext{
1 We remind that an equation of state is said "barotropic" if the pressure $P$ depends only on one variable, for instance the baryonic number density $n_{b}$.
} 
rotating relativistic NSs composed of neutrons, protons and electrons, with an "out-of-equilibrium" EOS. It should be kept in mind that in the following, we shall call "nonbarotropic" a peculiar type of nonbarotropic EOSs: the situation of npe matter with frozen composition.

The plan of the paper is as follows. In Section [II we start with an overview of the basic assumptions made in the study of the structure of cold neutron stars, with emphasis on the beta equilibrium between neutrons, protons and electrons, which constitute, for not too massive NSs, the most important part for studies of their oscillations: their core. We end this section with a description of our calculation of stationary rotating NSs and of the EOS used to get configurations to perturb in the following sections: the Prakash-Ainsworth-Lattimer (PAL) EOS 19]. In Section III] a short summary of the microphysics of "perturbed" NSs composed of npe matter is given. In this section, we justify the assumption of frozen composition, which is then used in Section [IV that describes the basis of the linear hydrodynamical study. The main feature of a nonbarotropic star is the existence of a nondegenerate spectrum for its polar modes, even when it is not rotating. As frozen composition results in a nonbarotropic effective EOS, these so-called gravity modes ( $g$-modes) are studied for the PAL EOS in Section $\square$ After which, in Section VI] we describe inertial modes of rotating NSs with the PAL EOS but without the (correct) hypothesis of frozen composition, before ending with gravity and inertial modes in rotating NSs taking into account the frozen composition. Finally, the results are discussed in the Conclusion.

\section{FULLY CATALYZED ROTATING RELATIVISTIC NEUTRON STARS}

\section{A. Model of the neutron star matter}

The outer layers of cold NSs are known to play key roles in several physical phenomena, as the early and late cooling of pulsars [20], the existence of glitches (21], 22], 23]) and the spectra of X-ray bursts 24]. Yet, since more than $95 \%$ of the mass of cold NSs is included in their core, outer layers are not relevant at the most basic levels of studies of both the structure of rotating relativistic stars and their oscillations. Thus, in the following, a NS is modeled by its core, and the outer layers are only discussed as time independent boundary conditions for the oscillations (see Section $\nabla$ ).

The minimal nontrivial description of a core of NS is a mixture of neutron, protons, and electrons at a temperature that is sufficiently low for the matter to be degenerate but sufficiently high for the nucleons not to be superfluid. Such conditions, which imply that thermal contributions to the EOS can be neglected, are satisfied for $10^{9} \mathrm{~K}<T<10^{10} \mathrm{~K}$, at densities above one tenth of normal nuclear density $n_{0} \widehat{=} 0.16 \mathrm{fm}^{-3}$. Moreover, when a NS has cooled down enough for these assumptions to be valid, the matter has become transparent for neutrinos, which therefore do not contribute to the EOS either.

Another physical constraint to take into account for the study of both unperturbed and perturbed stars is the electrical charge neutrality, which comes from the very short characteristic time scale of electromagnetic processes. In degenerate npe matter, the so-called plasma frequency of electrons, whose inverse give the typical time for the plasma to maintain charge neutrality, is

$$
\omega_{\mathrm{p} e}=\left(\frac{4 \pi e^{2} n_{e} c^{2}}{\mu_{e}}\right)^{1 / 2},
$$

where $\mu_{e}$ is the chemical potential of electrons similar to $\mu_{e} \sim 122\left(n_{e} / n_{0}\right)^{1 / 3} \mathrm{MeV}$ (see below), with the electron density $n_{e}$. This leads to an electromagnetic relaxation time $\tau_{\mathrm{pe}}=4 \times 10^{-22}\left(n_{e} / n_{0}\right)^{1 / 3} \mathrm{~s}$, which implies that, for our purpose (phenomena with typical time scales of some ms), matter can always be considered as neutral, and there is equality between the electron and proton densities $n_{e}=n_{p}$. This relation also makes thermodynamical quantities functions of nucleon (baryon) density $n_{b} \widehat{=} n_{n}+n_{p}$ and proton fraction $x_{p} \widehat{=} n_{p} / n_{b}$ only. Furthermore, at baryon densities of the order of $n_{0}$, nucleons form a strongly interacting Fermi system and matter is very rich in neutrons, while we can replace nucleon masses (which are assumed to be equal) by the neutron mass $m_{N}=m_{n}=939.57 \mathrm{MeV}$. If we neglect now the Coulomb interactions between charged constituents of matter and treat the electrons as a free ultrarelativistic Fermi gas, the energy per nucleon (excluding rest energy of nucleons) can be written

$$
E\left(n_{b}, x_{p}\right)=E_{\mathrm{N}}\left(n_{b}, x_{p}\right)+E_{e}\left(n_{b}, x_{p}\right),
$$

where $E_{\mathrm{N}}$ is the nucleon contribution, and the electron term $E_{e}$ is

$E_{e}\left(n_{b}, x_{p}\right)=\frac{3}{4} b u^{\frac{1}{3}} x_{p}^{\frac{4}{3}}, \quad b \widehat{=} \hbar c\left(3 \pi^{2} n_{0}\right)^{1 / 3}=331.4 \mathrm{MeV}$,

with $u \widehat{=} n_{b} / n_{0}$.

Extensive many-body calculations with realistic twonucleon and three-nucleon forces show that in the whole range of $0 \leq x_{p} \leq 1$, the energy per nucleon is (to a very good approximation) quadratic in the neutron excess parameter $\left(n_{n}-n_{p}\right) / n_{b}=1-2 x_{p}([25],[26])$. At $x_{p} \simeq 0.5$, characteristic value for terrestrial nuclear physics, this is just the consequence of the charge symmetry of stronginteraction. At $x_{p} \simeq 1$, this is a numerical fact. Therefore, the $x_{p}$-dependence factors out, and $E_{\mathrm{N}}$ is given by the formula

$$
E_{\mathrm{N}}\left(n_{b}, x_{p}\right)=W_{0}\left(n_{b}\right)+S\left(n_{b}\right)\left(1-2 x_{p}\right)^{2} .
$$

Here, $W_{0}\left(n_{b}\right)$ is the energy per nucleon of the symmetric nuclear matter $\left(x_{p}=\frac{1}{2}\right)$, and $S\left(n_{b}\right)$ is the symmetry 
energy at nucleon density $n_{b}$. Many possibilities exist for these two functions, among them those that form the PAL EOS 19]. This EOS, which is retained in this study, will be described in more detail with the presentation of the obtained background stars used for our modes calculations (Section 【D). However, to be able to calculate these unperturbed stars, another relation is needed to close the system of equations. This relation comes from the hypothesis of beta equilibrium between neutrons, protons and electrons.

\section{B. EOS in beta equilibrium}

For matter transparent to neutrinos, the equilibrium with respect to the weak-interaction beta-processes

$$
n \longrightarrow p+e+\bar{\nu}_{e}, \quad p+e \longrightarrow n+\nu_{e},
$$

implies a relation ${ }^{2}$ between the chemical potentials of the matter constituents, $\mu_{j} \widehat{=} \partial \rho / \partial n_{j}$, with

$$
\rho\left(n_{b}, x_{p}\right)=n_{b}\left(m_{N} c^{2}+E\right)
$$

the total energy density [energy per baryon $E$ that comes from Eq.(2) plus nucleon rest energies], and $j=n, p, e$. For matter transparent to neutrinos, this relation reads

$$
\mu_{n}=\mu_{p}+\mu_{e}
$$

With our quadratic form of the $x_{p}$-dependence of $E_{\mathrm{N}}$, Eq.(4), the beta equilibrium condition involves only the nuclear symmetry term. We have

$$
\mu_{n}-\mu_{p}-\mu_{e}=4 S\left(1-2 x_{p}\right)-b u^{\frac{1}{3}} x_{p}^{\frac{1}{3}}
$$

The resulting fraction of protons for matter in beta equilibrium, denoted by $x_{p}^{\mathrm{eq}}\left(n_{b}\right)$, is determined from

$$
\frac{x_{p}^{\mathrm{eq} \frac{1}{3}}}{1-2 x_{p}^{\mathrm{eq}}}=\frac{4 S}{b u^{\frac{1}{3}}}
$$

or

$$
y^{3}+\alpha y-\frac{1}{2}=0
$$

where $y^{3} \widehat{=} x_{p}^{\mathrm{eq}}$ and $\alpha\left(n_{b}\right) \widehat{=}\left(b u^{1 / 3}\right) /(8 S)$.

The last equation admits analytical solutions ( $c f$. Cardan's method) among which the only real one is

$x_{p}^{\mathrm{eq}}\left(n_{b}\right) \widehat{=} \frac{1}{2}-\frac{\alpha\left(n_{b}\right)}{2^{2 / 3}}\left[\left(\Gamma\left(n_{b}\right)+1\right)^{1 / 3}-\left(\Gamma\left(n_{b}\right)-1\right)^{1 / 3}\right]$

\footnotetext{
2 Note that this relation remains the same even when direct Urca processes, presented here, are not allowed due to kinematical reasons, and have to be replaced by processes that involve spectator nucleons, the modified Urca reactions.
}

with $\Gamma\left(n_{b}\right) \widehat{=} \sqrt{1+\frac{16}{27} \alpha\left(n_{b}\right)^{3}}$.

Then, the barotropic EOS in beta equilibrium, which is used for the calculation of unperturbed stars, is obtained from

$$
\rho_{\mathrm{eq}}\left(n_{b}\right)=\rho\left(n_{b}, x_{p}^{\mathrm{eq}}\left(n_{b}\right)\right),
$$

with the total pressure

$$
P_{\mathrm{eq}}\left(n_{b}\right)=\left.n_{b}^{2} \frac{\partial\left(\rho_{\mathrm{eq}} / n_{b}\right)}{\partial n_{b}}\right|_{x_{p}} .
$$

Note that when the chemical potential of the electrons is above the rest mass of muons, $m_{\mu} \sim 105.65 \mathrm{MeV}$, taking into account the appearance of the latter via the equilibrium between the processes

$$
e^{-} \rightarrow \mu^{-}+\nu_{e}+\bar{\nu}_{\mu} \text { and } \mu^{-} \rightarrow e^{-}+\bar{\nu}_{e}+\nu_{\mu}
$$

would have implied higher proton fractions. But as a rule, the role of muons is practically irrelevant, as far as the EOS is concerned, and we shall always neglect them.

\section{Stationary rotating relativistic neutron stars}

The numerical solution of the Einstein's equations for a cold stationary rotating relativistic star with a barotropic EOS, $P(\rho)$ [reached by Eq.(12) and (13)], has now become a classical problem (see [27] for a review) whose detail we will not enter into here. Let us just remind the reader of the main assumptions:

- there are two Killing vectors $\mathbf{k}$ (timelike at spacelike infinity) for stationarity and $\mathbf{m}$ (with closed orbits, spacelike everywhere except on the so-called rotation axis where it vanishes) for axisymmetry;

- spacetime is asymptotically flat, the metric $\mathbf{g}$ "tends toward" the flat Minkowski metric $\eta$ at infinity;

- matter is a perfect fluid whose energy-momentum tensor is ${ }^{3}$

$$
\mathbf{T} \widehat{=}(\rho+P) \mathbf{u} \otimes \mathbf{u}+P \mathbf{g},
$$

where $\mathbf{u}$ is the 4 -velocity of the fluid. Obviously, $P$ and $\rho$ are measured in the frame comoving with the fluid and thus are the equilibrium functions obtained in the previous section;

- the velocity field corresponds to rigid axisymmetric rotation and can be written

$$
\mathbf{u}=\lambda(\mathbf{k}+\Omega \mathbf{m})
$$

\footnotetext{
3 Except where otherwise noted we use natural units in which $c=\mathcal{G}_{\mathcal{N}}=1$.
} 
where $\Omega$ is by definition the angular velocity of the star and $\lambda$ a scalar field such as the norm of $\mathbf{u}$ is -1 (with our convention for the signature).

With these assumptions, the energy-momentum tensor satisfies the condition of circularity [28] and the maximal slicing quasi-isotropic coordinates [29] can be adopted to write the metric as

$$
\mathrm{d} s^{2} \widehat{=} g_{\mu \nu} \mathrm{d} x^{\mu} \mathrm{d} x^{\nu} \widehat{=}-\left(N^{2}-N_{\varphi} N^{\varphi}\right) \mathrm{dt}^{2}-2 N_{\varphi} \mathrm{dt} \mathrm{d} \varphi+\frac{A^{4}}{B^{2}}\left(\mathrm{dr} r^{2}+r^{2} \mathrm{~d} \vartheta^{2}+B^{4} r^{2} \sin ^{2} \vartheta \mathrm{d} \varphi^{2}\right)
$$

where we have introduced the notation of the $3+1$ formalism: $N$ is the lapse and $N^{\varphi}$ the third component of the shift 3-vector, with $N_{\varphi} \widehat{=} g_{\varphi i} N^{i}=A^{4} B^{2} N^{\varphi} r^{2} \sin ^{2} \vartheta$ the covariant $\varphi$-component of the latter.

Because of the existence of two Killing vectors all the functions only depend on $(r, \vartheta)$, and this choice of coordinates reduces the Einstein's equations to a system of elliptic equations that we solve with the fully relativistic spectral code presented in Bonazzola et al. 29]. This code also provides us with what will be the velocity field to perturb in the study of oscillations (Section IV]). Indeed the $\lambda$ scalar field, which normalizes the velocity, can be shown to satisfy

$$
\lambda=-\left(\left|\mathbf{k} \cdot \mathbf{k}+2 \Omega \mathbf{k} \cdot \mathbf{m}+\Omega^{2} \mathbf{m} \cdot \mathbf{m}\right|\right)^{-\frac{1}{2}},
$$

where - denotes the scalar product associated with the metric.

\section{Calculated background stars}

In order to study inertial modes of NSs more realistic than simple barotrops, we need an EOS for the nuclear matter that is also valid when the fluid is not in beta equilibrium (5). Indeed, as explained in the Introduction and as calculations will show in Section III a lump of matter transported out of its equilibrium position by an oscillation will not adjust its composition to its new environment instantaneously. This will require some time, which has to be taken into account in the dynamics since it allows new modes (see Section IV] to exist.

Among the numerous EOSs that describe nuclear matter in NSs, we decided to use the equation proposed by Prakash et al. [19] which is among the few which are valid also out of beta equilibrium. Moreover, this analytical EOS based partly on the experimental properties of nuclear matter, and partly on the results of many-body calculations of asymmetric nucleon matter, is in fact a set of EOSs. Indeed, several possibilities are given for the choice of the symmetry energy function $S\left(n_{b}\right)$, while the energy per nucleon of the symmetric nuclear matter $W_{0}\left(n_{b}\right)$ depends on some parameters. Thus, following Prakash et al., we have

$$
W_{0}\left(n_{b}\right) \widehat{=} \frac{3}{5} E_{F}^{(0)} u^{\frac{2}{3}}+\frac{1}{2} A u+\frac{B u^{\sigma}}{1+B^{\prime} u^{\sigma-1}}+3 \sum_{i=1,2} C_{i}\left(\frac{\Lambda_{i}}{p_{F}^{0}}\right)^{3}\left(\frac{p_{F}}{\Lambda_{i}}-\arctan \left[\frac{p_{F}}{\Lambda_{i}}\right]\right)
$$

where

- $E_{F}^{(0)}$ is the free nucleon gas Fermi energy at saturation density $(\sim 36 \mathrm{MeV})$;

- the dimensionless variable $u$ is $u \widehat{=} n_{b} / n_{0}$, where $n_{0}=0.16 \mathrm{fm}^{-3}$ is the saturation density of symmetric nuclear matter;

- the second and third terms, that depend on the constants $A, B, B^{\prime}$ and $\sigma$, reproduce the static inter- actions between nucleons and are chosen in such a way that the EOS is causal;

- the last two terms, that depend on the momentum, mimic the dynamical part of the strong interaction. In those terms, the $\Lambda$ parameters are characteristic lengths associated to coupling constants $C_{i}$. The first term (such as $C_{1}<0$ and $\Lambda_{1}=1.5 p_{F}{ }^{0}$ ) is attractive at large distances, whereas the second $\left(C_{2}>0\right.$ with $\left.\Lambda_{2}=3 p_{F}{ }^{0}\right)$ reproduces the re- 
pulsive behavior of the strong interaction at short scales.

In order to separate the kinetic and potential contributions in the symmetry energy, Prakash et al. 19] write

$$
S\left(n_{b}\right)=\left(2^{\frac{2}{3}}-1\right) \frac{3}{5} E_{F}^{(0)}\left(u^{\frac{2}{3}}-F(u)\right)+S_{0} F(u),
$$

where $F(u)$ is a function which describes the (poorly known) density dependence of $S$. To get $S\left(n_{0}\right)=S_{0}=30 \mathrm{MeV}$, one has $F(1)=1$.

Playing with the form of the functions $W_{0}\left(n_{b}\right)$ and $S\left(n_{b}\right)$ enables one to change the values of the compression modulus and the symmetry energy. However, this analytical EOS, that fits known results around the saturation density, has the drawback that, at low densities, it is no longer physical. Indeed, to be realistic at low densities, any EOS has to take into account the appearance of nuclei. Hence, for several choices of the key quantities (such as the value of the compression modulus and the symmetry energy) within the proposal of Prakash et al., pathological features like negative pressure or convective instability appear. Moreover, even for the EOSs that do not present these awkward characteristics, the low density part is unphysical and some prescription has to be adopted for the study of modes.

Among the functions and the set of parameters that Prakash et al. propose, we retain $F(u)=\sqrt{u}$ and take values of the parameters that give a compression modulus of either $180 \mathrm{MeV}$ (model A) or $240 \mathrm{MeV}$ (model B). In addition, to prevent the troubles linked to low densities, our choice is to cut the star below a given threshold density. As discussed in Section III since the mass of the crust is quite small, this cutoff does not really affect either the metric or the main properties of the star. Moreover, adding some boundary conditions on the surface described by the value of the density chosen for the cutoff is an easy way to mimic the phase transition that occurs between the outer-core and the inner-crust. This point will be discussed in more detail in the sections that deal with modes (Sections IV V VI) but it was noticed now to clarify Table【 Indeed, in this table are summarized the background configurations that we use. But in order to calibrate the effect of the cutoff on the modes, we tried several values for the density threshold. Hence, in Table \. we show structural properties of stars for the two EOSs that we keep with different central densities and the values that we take for the cutoff density ${ }^{4}: 0.08$ or 0.1 baryon. $\mathrm{fm}^{-3}$. As a first validation of the cutoff

\footnotetext{
4 i.e. one half or almost two thirds of the saturation density $n_{0}$. This range is consistent with realistic evaluations of the density at crust-core interface (see [30] and [31])
}

procedure, we can check that for given EOS and central density, the cutoffs imply differences in the gravitational mass of some few percent in the worst case. Finally, note also that the central (maximal) proton fraction is always below 1/9, which forbids direct Urca processes for npe matter (see also Section IIC).

\section{PERTURBED CONFIGURATIONS I: MICROPHYSICS}

\section{A. npe matter}

When trying to deal with modes of NSs in a not too unrealistic way, i.e. when trying to go beyond the simple model of a single barotropic fluid, the most basic model to consider is a NS composed of neutrons, protons and electrons. Remember that we deal here only with NSs sufficiently old to have become completely transparent to neutrinos, but we neglect possible superfluidity of nucleons. Anyway, even without neutrinos to complicate the game, these three fluids could, a priori, be only partially coupled and then three relativistic Euler equations (with coupling terms) could be needed ${ }^{5}$.

But, as discussed in Section III due to the very short time scale of the Coulomb interaction and due to its long range, it is well-known that, for oscillations with periods typical of NSs modes (some ms), protons and electrons are in fact interdependent with the same velocities and densities: matter is locally electrically neutral $x_{p}=x_{e}$. The first thing that we shall discuss now, is how the strong interaction between nucleons plays a similar but not exactly identical role. It makes the velocities of protons and neutrons equal (without superfluidity), while not leveling out their densities, as the latter depend on the weak-interaction (see Section IIB). As the demonstration of this in the most general case is quite tricky, we shall work here with a simpler situation that provides an order of magnitude estimate sufficient for our purpose.

\section{B. Damping of the relative $n-p$ flow}

We assume that there is no external force acting differently on $n$ and $p$ (i.e., we neglect the effects of the electromagnetic field). Consider the relative motion of neutrons and protons along the z-axis, with no baryon number flow, $v_{n}=-v_{p}=v$. Neglect all spatial gradients, and assume, that at $t=0$ we have $v(0)=v_{0}$. By solving the kinetic equations for neutron and proton

\footnotetext{
5 Notice that in this case, the easiest way to reach the equations of motion would not be to start with the usual conservation of the energy-momentum tensor, but to use the Lagrangian formalism for multifluids developed by Carter [32].
} 
TABLE I: Properties of the NSs that are used as backgrounds for the calculation of inertial and gravity modes. The entries in the table are: type of EOS (name of model); central relativistic enthalpy (dimensionless); central baryonic density (in fm ${ }^{-3}$ ); central proton fraction; density at the surface; enthalpy at the surface; gravitational mass (in solar mass units), circumferential equatorial radius (in $\mathrm{km}$ ) and compactness $\mathcal{C}=M_{\mathrm{G}} / R_{e q}$ (in dimensionless units). Note that Model $\mathrm{B}$ is stiffer and allows a minimal gravitational mass which is larger than $1.2 \mathrm{M}_{\odot}$. This is the reason why we do not have stars of model A and $\mathrm{B}$ with the same $1.2 \mathrm{M}_{\odot}$ mass, whereas we have stars of the same compactness $\mathrm{A}(26)$ and $\mathrm{B}(24)$, but also of the same $1.6 \mathrm{M}_{\odot}$ mass $\mathrm{A}(4)$ and $\mathrm{B}(3)$.

\begin{tabular}{|c|c|c|c|c|c|c|c|c|c|c|c|c|c|c|c|}
\hline EOS & \multicolumn{3}{|c|}{ Model A(21) } & \multicolumn{3}{|c|}{ Model A(26) } & \multicolumn{3}{|c|}{ Model A(4) } & \multicolumn{3}{|c|}{ Model B(24) } & \multicolumn{3}{|c|}{ Model B(3) } \\
\hline$h_{\mathrm{c}}$ & \multicolumn{3}{|c|}{0.21} & \multicolumn{3}{|c|}{0.26} & \multicolumn{3}{|c|}{0.4} & \multicolumn{3}{|c|}{0.24} & \multicolumn{3}{|c|}{0.3} \\
\hline$n_{\mathrm{c}}\left[\mathrm{fm}^{-3}\right]$ & \multicolumn{3}{|c|}{0.573} & & 0.676 & & \multicolumn{3}{|c|}{0.966} & \multicolumn{3}{|c|}{0.540} & \multicolumn{3}{|c|}{0.635} \\
\hline$x_{p_{\mathrm{C}}}[\%]$ & \multicolumn{3}{|c|}{7.43} & & 8.05 & & \multicolumn{3}{|c|}{9.47} & & 7.22 & & \multicolumn{3}{|c|}{7.81} \\
\hline$n_{\text {surf }}\left[\mathrm{fm}^{-3}\right]$ & 0 & 0.08 & 0.1 & 0 & 0.08 & 0.1 & 0 & 0.08 & 0.1 & 0 & 0.08 & 0.1 & 0 & 0.08 & 0.1 \\
\hline$h_{\text {surf }}\left[10^{-2}\right]$ & 0 & 2.0 & 2.3 & 0 & 2.0 & 2.3 & 0 & 2.0 & 2.3 & 0 & 1.9 & 2.2 & 0 & 1.9 & 2.2 \\
\hline$M_{\mathrm{G}}\left[M_{\odot}\right]$ & 1.20 & 1.18 & 1.17 & 1.35 & 1.34 & 1.33 & 1.60 & 1.591 & 1.59 & 1.42 & 1.41 & 1.40 & 1.60 & 1.59 & 1.58 \\
\hline$R_{e q}[\mathrm{~km}]$ & 11.3 & 10.4 & 10.3 & 11.1 & 10.4 & 10.3 & 10.2 & 10.0 & 9.9 & 11.8 & 11.1 & 11.0 & 11.6 & 11.0 & 10.9 \\
\hline $\mathcal{C}\left[10^{-1}\right]$ & 1.57 & 1.67 & 1.68 & 1.79 & 1.91 & 1.91 & 2.33 & 2.362 & 2.38 & 1.77 & 1.88 & 1.89 & 2.03 & 2.13 & 2.14 \\
\hline
\end{tabular}

distribution functions, with a collision term due to the strong-interaction nucleon-nucleon scattering, we will find that $v(t)=v(0) e^{-t / \tau_{n p}}$, where $\tau_{n p}$ is the relaxation time after which the relative $n-p$ flow is damped. As a nucleon fluid forms a strongly interacting dense Fermi liquid, the $n-p$ scattering cross section to be used in the kinetic equation is different from that in vacuum, and the transport process refers to the quasiparticles of the Fermi liquid theory (see, e.g., Baym et al. [21]).

For symmetric nuclear matter at normal nuclear density, one gets, using the Fermi liquid theory, $\tau_{n p} \sim 10^{-18} T_{9}^{-2} \mathrm{~s}$ (Haensel 33$]$ ), where $T_{9}$ is the temperature in $10^{9} \mathrm{~K}$. For a highly asymmetric neutron star matter, with $x_{p}=0.05$, simple estimate in which the vacuum $n-p$ scattering cross sections are used, were obtained by Baym et al. [21], $\tau_{n p} \sim 10^{-19} T_{9}^{-2}$ s. Hence, in view of (microscopic) smallness of $\tau_{n p}$ compared to any other time scales relevant for our study, neutrons and protons can be assumed to move together as one single nucleon fluid. Yet, as already mentioned, the ratio between their densities is not governed by strong interaction but by weak-interaction, which makes their relation different from the relation between electrons and protons as we shall see now.

\section{Nonequilibrium beta-processes}

Since the time for beta equilibrium (5) to settle in depends on the production of thermal neutrinos, for the time being we shall consider an element of hot npe matter at temperature $T$. This lump has an instantaneous nucleon density $n_{b}=n_{p}+n_{n}$, which due to its temporal evolution (that results from the matter flow) is out of beta equilibrium, so that

$$
\delta \mu \widehat{=} \mu_{p}+\mu_{e}-\mu_{n} \neq 0
$$

Let us also characterize this deviation from beta equilibrium by $\xi \widehat{=} x_{p}-x_{p}^{\mathrm{eq}}\left(n_{b}\right)$.

Each of the three fluids (neutrons, protons and electrons), is separately in thermodynamic equilibrium, due to strong and electromagnetic interactions. However, weak-interaction processes may be too slow to establish beta equilibrium at $\left[n_{b}, T\right]$. We denote the values of $n_{n}$ and $n_{p}$, which correspond to beta equilibrium at $\left[n_{b}, T\right]$, by $n_{n}^{\mathrm{eq}}, n_{p}^{\mathrm{eq}}$. The values $n_{n}^{\mathrm{eq}}, n_{p}^{\mathrm{eq}}$ are the reference ones, for a given $\left[n_{b}, T\right]$ pair, and are compared with actual values $n_{n}, n_{p}$.

A nonzero value of $\delta \mu$, Eq. (21), implies nonequilibrium reactions, which tend to decrease deviation from beta equilibrium (in accordance with the Le Châtelier principle). Let us denote the rate of the change of $n_{p}$ at fixed $n_{b}$ by $\Delta \Gamma\left(n_{b}, T, \delta \mu\right)$ (see Sect. 3.5 of Yakovlev et al. 20]). We then have

$$
\begin{gathered}
\Delta \Gamma\left(n_{b}, T, \delta \mu=0\right)=0, \\
\Delta \Gamma\left(n_{b}, T,-\delta \mu\right)=-\Delta \Gamma\left(n_{b}, T, \delta \mu\right),
\end{gathered}
$$

while at fixed $n_{b}$, we can write

$$
\dot{n}_{p}=-\dot{n}_{n}=\dot{\xi} n_{b}=\Delta \Gamma\left(n_{b}, T, \delta \mu\right),
$$

where the dots denote time derivatives.

In the linear approximation, $\xi \ll x_{p}^{\mathrm{eq}}, \delta \mu$ implied by $\xi$ at a fixed $n_{b}$ is

$$
(\delta \mu)_{n_{b}}=\left(\frac{\partial \delta \mu}{\partial x}\right)_{n_{b}} \xi=\frac{4}{3} S\left(4+\frac{1}{x}\right) \xi,
$$

where all coefficients are calculated at $n_{b}, x_{p}^{\mathrm{eq}}$.

In what follows, we assume in addition that $\delta \mu /(\pi k T) \ll 1$, which is equivalent to 
$\delta \mu / \mathrm{MeV} \ll 0.27 T_{9}$. This means that matter deviates only weakly from the beta equilibrium, an approximation which is valid for small amplitude of neutron star pulsation. Moreover, we remind the reader that nucleons are supposed to be normal (no superfluidity, valid for $T_{9}>1$ ) and we will consider here only cases with $x_{p}<1 / 9$, which is in perfect agreement with our background models (see Table I), so that only modified Urca processes are allowed (see Lattimer et al. 34]). Notice that the case of direct Urca processes would anyway lead to a similar conclusion, just changing quantitatively the result. Under the previous assumptions, the formula for $\Delta \Gamma$ reads, keeping only terms linear in $\delta \mu$,

$\Delta \Gamma\left(n_{b}, T, \delta \mu\right)=3.9 \times 10^{28}\left(\frac{n_{p}}{n_{0}}\right)^{1 / 3} T_{9}^{6} \frac{\delta \mu}{1 \mathrm{MeV}} \mathrm{cm}^{-3} \mathrm{~s}^{-1}$

where normal nuclear density $n_{0}=0.16 \mathrm{fm}^{-3}$.

In the relaxation time approximation, we can rewrite the formula for $\Delta \Gamma$ as

$$
\Delta \Gamma=-\frac{n_{p}-n_{p}^{\mathrm{eq}}}{\tau_{\beta}} .
$$

At fixed $n_{b}$, relaxation from a state initially off equilibrium by $\delta n_{p}^{0} \equiv n_{p}^{0}-n_{p}^{\mathrm{eq}}$, towards beta equilibrium, proceeds according to

$$
n_{p}(t)=n_{p}^{\mathrm{eq}}+\delta n_{p}^{0} \mathrm{e}^{-t / \tau_{\beta}} .
$$

Using the relation between $\delta \mu$ and $\delta n_{p}$, one shows that the beta-relaxation time is given by

$$
\tau_{\beta}=5.1 \times 10^{5}\left(T_{9}\right)^{-6}\left(\frac{n_{b}}{n_{0}} \cdot \frac{x_{p}}{0.01}\right)^{1 / 3} \mathrm{~s} .
$$

Under the prevailing conditions, this time scale is so long compared to other typical time scales that it is a very good assumption to consider that every lump of matter keeps a frozen composition (value of $x_{p}$ ) in perturbed configurations $^{6}$. In Section [IV we shall see how, from the hydrodynamical point of view, this condition can be taken into account and leads to a nonbarotropic equation of state. Before that, we shall briefly discuss for npe matter viscosity coefficients and viscous damping times of oscillations.

\footnotetext{
${ }^{6}$ If hyperons were included, they would also move together with the bulk matter due to the strong interaction, but concerning the respective ratios, the situation would be a little different due to nonleptonic strangeness violating reactions. These weakinteraction reactions without leptons like
}

$$
\mathrm{N} \Lambda \rightleftharpoons \mathrm{Nn}, \quad \mathrm{nn} \rightleftharpoons \mathrm{p} \Sigma^{-},
$$

where $\mathrm{N}$ is a nucleon, have relaxation times of the order of the millisecond for $T \sim 10^{9} \mathrm{~K}([35],[36])$. Thus the composition could be no longer frozen for millisecond oscillations.

\section{Viscosity of npe matter}

As we shall see later (Sections $\nabla$ and $\nabla \mathbf{~ I ) , ~ w e ~ d e a l ~}$ in this study only with rotational and gravity $m=2$ modes, with the idea of focusing on the gravitational driven instability of the $r$-modes. For them, it is wellknown [5] that shear viscosity kills the CFS instability (see Section VI) for low temperatures, whereas bulkviscosity does it for high temperatures. On the other hand, gravity modes of cold NS star are not directly relevant for gravitational waves emission due to their very weak coupling with the gravitational field. Hence our goal here is just to "verify" that to use the equations of motion for perfect fluids is quite reasonable for the durations of our simulations (of the order of 1 second) during which viscosity would not really have time to damp the modes.

The dissipation connected with viscosity of the npe matter is characterized by two density and temperature dependent parameters, shear viscosity $\eta$ and bulkviscosity $\zeta$. Shear viscosity results from the momentum transfer in the scattering processes between the npe matter constituents. It is mostly determined by largemomentum transfer collisions, and therefore the dominant contribution to $\eta$ is that of nonsuperfluid neutrons. An approximate analytic expression describing results obtained by Flowers \& Itoh [37] is

$$
\eta \simeq \eta_{n} \simeq 1.6 \times 10^{16}\left(\rho_{14}\right)^{2}\left(T_{9}\right)^{-2} \mathrm{~g} \mathrm{~cm}^{-1} \mathrm{~s}^{-1},
$$

where $\rho_{14} \widehat{=} \rho / 10^{14} \mathrm{~g} \mathrm{~cm}^{-3}$.

On the other hand, bulk-viscosity results from the nonequilibrium beta-processes previously discussed. But remember that for the notion of bulk-viscosity to be valid, the deviations from the beta equilibrium measured by $\delta \mu$ should be much smaller than $k T$. In such conditions, by considering periodic pulsations of local pressure in the npe matter, one can obtain an expression for the mean heat deposition due to the nonequilibrium processes. When the relation between Fermi momenta

$$
p_{\mathrm{F} n}>p_{\mathrm{F} p}+p_{\mathrm{F} e}
$$

is fulfilled, which in the case of npe matter corresponds to $x_{p}<1 / 9$, the direct Urca processes are prohibited. As already mentioned above with the description of our background stars, this is always the case in this study (see Table 凹). Hence here only modified Urca (mUrca) processes are allowed, with their neutron branch

$$
\begin{gathered}
n+n \longrightarrow n+p+e+\bar{\nu}_{e}, \\
n+p+e \longrightarrow n+n+\nu_{e},
\end{gathered}
$$

and their proton branch

$$
\begin{gathered}
p+n \longrightarrow p+p+e+\bar{\nu}_{e} \\
p+p+e \longrightarrow p+n+\nu_{e} .
\end{gathered}
$$


The formulae obtained by Haensel et al. (see [38], 39] for more detail) and applied to the npe matter give for the resulting bulk-viscosity

$$
\zeta_{n}^{\mathrm{mUrca}} \sim 10^{20} \omega_{4}^{-2}\left(T_{9}\right)^{6} \mathrm{~g} \mathrm{~cm}^{-1} \mathrm{~s}^{-1} .
$$

Here, $\omega_{4}$ is the angular frequency of pulsations measured in the units of $10^{4} \mathrm{~s}^{-1}$. Even if we shall not need it here, let us mention that the magnitude of the bulk-viscosity increases by many orders if the direct Urca (dUrca) processes are allowed. These processes lead to

$$
\zeta_{n}^{\mathrm{dUrca}} \sim 10^{25} \omega_{4}^{-2}\left(T_{9}\right)^{4} \Theta_{n p e} \mathrm{~g} \mathrm{~cm}^{-1} \mathrm{~s}^{-1},
$$

where the threshold factor $\Theta_{\text {npe }}$ vanishes if $p_{\mathrm{F} n}>p_{\mathrm{F} p}+p_{\mathrm{Fe}}$.

With Eq.(30), we obtain the time scale for the shear viscosity [40]

$$
\tau_{\eta}=\alpha_{\text {mode }}^{\eta} \frac{\rho R^{2}}{\eta} \simeq \alpha_{\text {mode }}^{\eta} \frac{R_{6}^{2} T_{9}^{2}}{\rho_{15}} \times 10^{9} \mathrm{~s},
$$

where $R=R_{6} \times 10^{6} \mathrm{~cm}$ is the core radius, $\rho=\rho_{15} \times 10^{15} \mathrm{~g} \mathrm{~cm}^{-3}$ the "mean" core density, and $\alpha_{\text {mode }}^{\eta}$ a numerical coefficient that depends on the pulsational mode.

Eq. (35) give a very rough estimate of the bulk-viscosity damping time scale due to mUrca bulk-viscosity:

$$
\tau_{\zeta}^{\mathrm{mU}} \sim \alpha_{\text {mode }}^{\zeta, \mathrm{mU}} \frac{\rho R^{2}}{\zeta^{\mathrm{mU}}} \simeq \alpha_{\text {mode }}^{\zeta, \mathrm{mU}} \frac{R_{6}^{2}}{T_{9}^{6}} \times 10^{8} \mathrm{~s} .
$$

We see with these formulae that around $10^{9} \mathrm{~K}$, none of the viscosities damps the modes very quickly ${ }^{7}$. More precisely, for $T_{9}=5$ the damping time scale is of the order of hours. Hence for an evolution lasting a few seconds the assumption of perfect fluid is not so bad.

As a final remark, let us mention that, in a star of radius $R$, the presence of a core of radius $R_{D}$ where the direct Urca process is allowed would imply a damping time scale given by

$$
\tau_{\zeta}^{\mathrm{dU}}=\alpha_{\text {mode }}^{\zeta, \mathrm{dU}} \frac{\rho R^{2}}{\zeta^{\mathrm{dU}}} \simeq \alpha_{\text {mode }}^{\zeta, \mathrm{dUra}} \frac{R_{6}^{2}}{T_{9}^{4}} \times 10^{3} \mathrm{~s},
$$

with, for the $r$-modes, $\alpha^{\zeta, \mathrm{dU}}$ much greater than one (see [41] for the case of the CFS instability). Additional increase of $\alpha_{\text {mode }}^{\zeta, \text { dUra }}$ results from the fact that the sphere in which energy is dissipated has $R_{D}$ for radius, that is only a fraction of the stellar radius $R$ in which the mode propagate. However, the direct Urca process would not be for the modes as dramatic as the presence of hyperons and at $T_{9}=5$ we would still expect damping on a time scale of minutes.

\footnotetext{
7 the expected value of $\alpha^{\zeta, \mathrm{mU}}$ for the $r$-mode is significantly greater than one.
}

\section{PERTURBED CONFIGURATIONS II: HYDRODYNAMICS}

\section{A. Assumptions for the linear hydrodynamical study}

Doing time evolutions in general relativity is much more complicated than the solution of Einstein's equations for stationary and axisymmetric configurations. Hence, it is natural (at least in a first study) to begin with some approximations in order to simplify the problem. Thus, for the rest of this work, we assume that

- the perturbed star is "not too far" (see 7] for a proper definition of this) from the unperturbed one. It is then legitimate to linearize all equations (that govern both the material fields and the gravitational field) with respect to a parameter that indexes a continuous family of time dependent solutions and whose value is 0 for the unperturbed configuration. Obviously, this approximation means that in the following study of modes, the zero order terms are input that come from the unperturbed calculation presented in Section IID

- the Eulerian perturbations of the gravitational field (i.e. of the metric) are quite "small" and not fundamental for the physics. We then neglect them and write

$$
\delta g_{\mu \nu} \equiv 0,
$$

where $\delta g_{\mu \nu}$ are the Eulerian perturbations of the metric $g_{\mu \nu}$. This is the so-called relativistic Cowling 42 approximation, introduced by Mc Dermott et al. 43] in the study of oscillations of warm neutron stars. As discussed by several authors, this approximation has several drawbacks in the relativistic case $([9],[44])$, but it was also shown that it does not change dramatically the main feature of inertial modes (see [5] for a review). In addition, implementing the evolution of some metric perturbations (see Section VI) in our code would require a non-negligible amount of work since boundary conditions have to be treated carefully with spectral methods [45];

- the NS is slowly rotating. Only terms linear in the angular velocity $\Omega$ are kept in the equations, and every zero order term is assumed to only depend on the radial coordinate. This assumption was discussed in Paper I, and we shall stress here just one point : its use makes it sufficient to work with Hartle's equations [46] and not with the full Einstein's equations for the unperturbed calculation. Yet, as we plan to get rid of it in the future and since we have a fully relativistic code, it was easier for us to use this code to get the zero order terms. Moreover, we verified in this way the relevance of the 
slow rotation approximation. For more detail, see Paper I;

- the spatial part of the metric is conformally flat (Isenberg-Wilson-Mathews approximation, 47], [48] and [4]), i.e. the metric (17) becomes

$\mathrm{ds}^{2}=-\left(N^{2}-N_{\varphi} N^{\varphi}\right) \mathrm{dt}^{2}-2 N_{\varphi} \mathrm{dt} \mathrm{d} \varphi+h_{i j} \mathrm{~d} x^{i} \mathrm{~d} x^{j}$

with $i, j \in(1,2,3)$ and

$$
\mathbf{h} \equiv a \eta,
$$

where $a$ is the conformal factor and $\eta$ the flat Eu- clidean 3-metric. The conformal approximation was shown to be very good for (even fast) rotating isolated NSs by Cook et al. [50], and once again using the fully relativistic code we could verify this before making the assumption in the linear part of the work. Moreover, as we already use the Cowling approximation, we do not really care to "kill again" the GW content of spacetime, which can at a first level be obtained with some "post-Newtonian multipolar scheme" (see for instance [51]).

With all these assumptions, the final frozen metric used in the first order calculations is written

$$
\mathrm{ds}^{2} \widehat{=}-\left(N^{2}-a^{2} r^{2} \sin [\vartheta]^{2} N^{\varphi} 2\right) \mathrm{dt}^{2}-2 a^{2} r^{2} \sin [\vartheta]^{2} N^{\varphi} \mathrm{dtd} \varphi+a^{2} \mathrm{dl}^{2},
$$

where any function $N, N^{\varphi}$ and $a$ only depends on the radial coordinate $x^{1} \widehat{=} r$, while $\mathrm{dl}^{2}$ is the length interval in the flat 3 -space. With this metric, the linearized equations of motion can be easily written in a way similar to the Newtonian equations.

\section{B. Equations of motion}

For reasons described in Section III we assume that there is only one fluid whose relativistic equations of motion are obtained by the usual conservation of the energymomentum tensor of a perfect fluid

$$
\nabla \cdot \mathbf{T}=0
$$

with

$$
\mathbf{T} \widehat{=}(\rho+P) \mathbf{u} \otimes \mathbf{u}+P \mathbf{g},
$$

where $\nabla$ is the covariant derivative associated with the Levi-Civita affine connection for the given metric (42).
For the isentropic motion of a perfect fluid with a given EOS, it is well-known that, due to thermodynamics, the system of equations formed by the baryonic number conservation and the energy-momentum conservation (43) is degenerate. Hence, we shall work with the baryonic number conservation,

$$
\nabla \cdot\left(n_{b} \mathbf{u}\right)=0
$$

where $n_{b}$ is the baryonic density measured in the fluid's local rest frame, plus the projections on the 3-space of the relativistic Euler equations (EE) obtained from Eq. (43). More precisely, the useful equations are those reached after linearizing close to a solution that describes the rigidly rotating star, Eqs. (1618).

As was briefly described in Paper I, in order to get equations as similar as possible to the Newtonian EE, we use the well-known results about rigid rotation of relativistic stars, Eqs. (16/18), and write the perturbed 4 -velocity as

$$
u^{\mu}[t, r, \vartheta, \phi]=\frac{1}{N[r]} \mid \begin{aligned}
& 1+\delta U^{0}[t, r, \vartheta, \phi] \\
& (N[r] / a[r])^{2} \delta U^{r}[t, r, \vartheta, \phi] \\
& (N[r] / a[r])^{2} \delta U^{\vartheta}[t, r, \vartheta, \phi] / r \\
& \Omega+(N[r] / a[r])^{2} \delta U^{\varphi}[t, r, \vartheta, \phi] /(r \sin [\vartheta])
\end{aligned}
$$

Here, $\delta U^{0}, \delta U^{r}, \delta U^{\vartheta}$ and $\delta U^{\varphi}$ are first order quantities added to the unperturbed 4 -velocity that describes rigid rotation, with $\delta U^{0}$ that is not a dynamical variable but determined according to the constraint that $\mathbf{u}$ is a 
4-velocity : $\mathbf{u} \cdot \mathbf{u}=-1$ for the chosen signature of the metric. Furthermore, $\delta U^{r}, \delta U^{\vartheta}$ and $\delta U^{\varphi}$ are not contravariant components of a 4 -vector but convenient variables used in our calculations, which are nevertheless the components of $\vec{W}$, a 3-vector, in the orthonormal basis associated with the spherical system of coordinates for the flat 3 -space. With all this, we obtain

$$
\begin{gathered}
\left(\partial_{t}+\Omega \partial_{\phi}\right) \delta U^{r}+\delta\left[\partial_{r} P / f\right]-2 \delta U^{\varphi} \sin [\vartheta]\left(\left(\Omega-N^{\varphi}\right)\left(r a^{\prime} / a+1-r N^{\prime} / N\right)-r N^{\varphi^{\prime}} / 2\right)=0, \\
\left(\partial_{t}+\Omega \partial_{\phi}\right) \delta U^{\vartheta}+\delta\left[\partial_{\vartheta} P /(r f)\right]-2 \delta U^{\varphi} \cos [\vartheta]\left(\Omega-N^{\varphi}\right)=0, \\
\left(\partial_{t}+\Omega \partial_{\phi}\right) \delta U^{\varphi}+\delta\left[\partial_{\varphi} P /(f r \sin [\vartheta])\right]+2 \delta U^{r} \sin [\vartheta]\left(\left(\Omega-N^{\varphi}\right)\left(r a^{\prime} / a+1-r N^{\prime} / N\right)-r N^{\varphi^{\prime}} / 2\right) \\
+2 \delta U^{\vartheta} \cos [\vartheta]\left(\Omega-N^{\varphi}\right)=0,
\end{gathered}
$$

where the ' indicates the derivation with respect to the radial coordinate, $\delta$ is the operator for the Eulerian perturbation and $f \widehat{=} \rho+P$.

To this system of equations, we add the linearized baryon number conservation that, in the slow rotation approximation, can be written as

$$
\left(\partial_{t}+\Omega \partial_{\varphi}\right) \delta \tilde{n}+\frac{N^{2}}{a^{2}} \operatorname{div}(\tilde{n} \vec{W})=0,
$$

where we define $\tilde{n} \widehat{=} n_{b} N[r]^{2} a[r]$ and introduce the already mentioned 3 -vector $\vec{W}$, using also the usual 3-dimensional spatial divergence operator.

Hence, with the introduction of the relativistic anelastic approximation defined in Paper I (see also this paper for a discussion of the motivations for this approximation), we finally get the Newtonian-like equation

$$
\operatorname{div}(\tilde{n} \vec{W})=0
$$

which is the equation used in the current version of the code. The only missing element to close the system of equations is now the way thermodynamical quantities (mainly the pressure) are perturbed, i.e. we need to give a prescription to calculate in system (47) the term

$$
\delta\left[\frac{1}{f} \vec{\nabla} P\right] .
$$

This prescription will come directly from the previous analysis and from the fact that matter keeps a frozen composition when it is perturbed.

\section{Motion of perturbed npe matter}

Let us first summarize the conclusions that come from the microphysical study overviewed in Section 11
- the unperturbed star is described by an effective barotropic EOS with the npe matter in beta equilibrium;

- the perturbed (oscillating) star can not be described by the same EOS since matter is no longer in beta equilibrium;

- the easiest but most general EOS for perturbed npe matter at low temperature depends on two parameters: the baryonic density $n_{b}$ and the proton fraction $x_{p}$;

- for this nonbarotropic EOS and in the situation retained for the present study, the physical conditions in which the perturbations occur are that a given piece of npe matter will move with a frozen composition that corresponds to its composition in the unperturbed situation.

This last point needs now to be translated into an equation that will replace the beta equilibrium relation in the case of perturbed matter. But before we make it more explicit, we shall remind what turns out to be the situation when instantaneous beta equilibrium (or barotropicity) is assumed. This situation was supposed to occur in Paper I, and is still useful here since we have tried to modify our algorithms as little as possible.

In this case, thermodynamics tells us that both the pressure $P$ and the total energy density $\rho$ are functions only of the baryonic density $n_{b}$. Hence, the term (50) can be written as the Eulerian perturbation of an exact derivative (or the gradient of a scalar field), or equivalently as the gradient of the perturbation of a field that comes from an exact derivative, the relativistic enthalpy

$$
\delta\left[\frac{1}{f\left(n_{b}\right)} \vec{\nabla} P\left(n_{b}\right)\right] \widehat{=} \delta \vec{\nabla} H\left(n_{b}\right) \equiv \vec{\nabla} \delta H\left(n_{b}\right) .
$$


Using this new function, one can write the $\mathrm{EE}$ in a condensed and well-known form, which makes the system self-consistent and which was described in Paper I.

When we can no longer use the same EOS for the perturbed star and the background star, the previous list of conclusions are translated into equations in the following ways. First, as already mentioned, they imply that we need an EOS for the perturbed fluid that relates the perturbation of the pressure $P$ and energy density $\rho$ with (for instance) the perturbation of the baryonic density $n_{b}$ and the perturbation of the proton fraction $x_{p}$

$$
\delta P\left(n_{b}, x_{p}\right)=\left.\frac{\partial P}{\partial n_{b}}\right|_{x_{p}} \delta n_{b}+\left.\frac{\partial P}{\partial x_{p}}\right|_{n_{b}} \delta x_{p},
$$

and no longer only with the first one ${ }^{8}$

$$
\left.\delta P_{\beta}\left(n_{b}\right) \widehat{=} \frac{\mathrm{d} P}{\mathrm{~d} n_{b}}\right|_{x_{p} \equiv x_{p}^{\mathrm{eq}}\left(n_{b}\right)} \delta n_{b} \widehat{=} \frac{P}{n_{b}} \gamma_{\beta} \delta n_{b},
$$

as it was when instantaneous beta equilibrium and a barotropic EOS were assumed. Note that here we have introduced the adiabatic index for the matter in beta equilibrium, $\gamma_{\beta}$. This index will be used in the following and is a priori not a constant (as it is in the polytropic case). Moreover, the function $x_{p}^{\mathrm{eq}}\left(n_{b}\right)$, which links the proton fraction with the baryonic density for npe matter in beta equilibrium, is of course the result (11) obtained in Section II

One of the main implications of an EOS dependent on two parameters is that it makes it impossible to define the relativistic enthalpy by the relation (51), since the fraction is no longer an exact derivative. Hence, the term (50) can no longer be regarded as a function of a single variable and the system has to be completed by an additional equation to be closed, an equation that describes the fact that the matter has a frozen composition.

This prescription means that the Lagrangian perturbation of the proton fraction is 0 (as far as the EE are con-

\footnotetext{
8 From the relativistic point of view, it is crucial to verify that the employed EOS dependent on these parameters is covariant. It can be shown to be trivial if we write the numbers $n_{b}$ and $x_{p}$ as Lorentz scalars defined with the baryon, neutron and proton 4-currents. It gives

$$
n_{i} \widehat{=} \sqrt{-\mathbf{n}_{\mathbf{i}} \cdot \mathbf{n}_{\mathbf{i}}}
$$

where $i$ is either $b, n$ or $p$. Moreover, with the relations between 4-currents and 4-velocities

$$
\mathbf{n}_{\mathbf{i}}=n_{i} \mathbf{u}_{\mathbf{i}}
$$

it is easy to see that assuming each of the velocity fields to be equal implies that the most general EOS can only depend on two Lorentz scalars, $n_{b}$ and $x_{p}$ for instance.
}

cerned) or (equivalently) that its derivative along the velocity field vanishes. Addressing with $\Delta$ the Lagrangian perturbation of any quantity, we then have for the motions that we are dealing with

$$
\Delta P=\frac{P}{n_{b}} \gamma_{F} \Delta n_{b},
$$

where

$$
\left.\gamma_{F} \widehat{=} \frac{\partial \ln [P]}{\partial \ln \left[n_{b}\right]}\right|_{x_{p}}
$$

is the adiabatic index for a frozen composition. Furthermore, the formal relation, which links Lagrangian and Eulerian perturbations by the Lie derivative with respect to the Lagrangian deplacement $\xi$,

$$
\Delta-\delta \widehat{=} £_{\xi}
$$

enables us to arrive to the final expression

$$
\delta P=\delta P_{\beta}+\frac{P}{n_{b}} \Delta n_{b}\left(\gamma_{F}-\gamma_{\beta}\right)
$$

where the first part of the perturbation is what we would get if beta equilibrium was instantaneous. A similar expression can be found for the perturbation of $f \widehat{=} \rho+P$ with some " $\zeta$ " coefficients instead of the $\gamma$. Nevertheless, the relevant quantity at the linear order for matter close to beta equilibrium is

$$
\zeta_{F}-\zeta_{\beta}=\frac{P}{f}\left(\gamma_{F}-\gamma_{\beta}\right) .
$$

The expression (59) and its equivalent for $f$ are those we use in the EE in order to write the term (50) as the perturbation of the gradient of the enthalpy plus a contribution that exists only for nonbarotropic stars. In this way, we do not need to change our algorithms (see Paper I for more detail) for the solution of the EE and the anelastic equation (49), whereas we only need to add, as a source term, the second part of these expressions. Moreover, written like this, this source term depends only on the Lagrangian perturbation of the baryonic density $\Delta n_{b}$ (as far as first order terms are concerned), since we have

$$
\begin{aligned}
\delta\left[\frac{1}{f} \vec{\nabla} P\right]= & \vec{\nabla} \delta H+\frac{P}{f} \vec{\nabla}\left(\frac{\Delta n_{b}}{n_{b}}\left(\gamma_{F}-\gamma_{\beta}\right)\right) \\
& +\vec{\nabla} H \frac{\Delta n_{b}}{n_{b}}\left(\gamma_{F}-\gamma_{\beta}\right)\left(1-\frac{P}{f}\right),
\end{aligned}
$$

in which $\Delta n_{b}$ is obtained by solving the equation

$$
£_{\mathbf{u}} x_{p}=0,
$$

where $\mathbf{u}$ is the 4 -velocity. Indeed, when we linearize this equation and use the anelastic approximation, we get after some algebra the Newtonian-like advection equation for $\Delta n_{b}$

$$
\left(\partial_{t}+\Omega \partial_{\varphi}\right) \Delta n_{b}=\frac{N^{2}}{a^{2}} \vec{W} \cdot \vec{\nabla} n_{b}
$$


where the usual Newtonian notations were used. This new equation enables the existence of modes related to the gradient of composition and whose restoring force is gravitation. These $g$-modes for cold but heterogeneous NSs were first discussed by Reisenegger \& Goldreich 52 and will be the subject of the next section.

\section{GRAVITY MODES}

\section{A. General features of composition gravity modes}

Before dealing with the more complex situation of the modes of a stratified rotating relativistic star, it is worth to recall the situation for the modes of a nonrotating star, in order to better recognize in the following their scions. With the already mentioned assumptions, it is quite simple to describe. We consider only the npe core of NSs, without superfluidity, without magnetic field and without trying to follow modes of the crust. Hence due to the spherical symmetry, only polar fluid modes $^{9}$ can exist and the spectrum of axial modes is degenerate at zero frequency. Moreover, using the relativistic generalization of the anelastic approximation [cf. Eq.(49), Paper I where it was introduced and also the discussion in the Appendix], the only hydrodynamical modes left in the nonrotating cold star are low-frequency modes whose restoring force is gravity, the so-called composition $g$-modes. Indeed, the main consequence of the anelastic approximation is to filter out pressure modes (see the Appendix). In addition, the relativistic Cowling approximation prevents us from having $w$-modes (some of which could have been axial), which are mainly oscillations of the spacetime itself.

Gravity modes of NSs have already been the subject of several works, even if they were first thought to be degenerate at zero frequency due to beta equilibrium (Thorne, 53]). Thus, gravity modes that exist because of the presence of temperature gradients in warm NSs were studied by Mc Dermott et al. [43], who introduced the relativistic Cowling approximation. They found that the thermal $g$-modes were concentrated into a thin layer close to the surface, as was the case in the analysis of Finn [54] on gravity modes linked to discrete changes in composition of the crust. The first study considering Newtonian gravity modes associated with smooth composition gradients in the core of NSs is that of Reisenegger \& Goldreich 52]. However, they neglect the effect of the strong interaction, which is inappropriate since nucleons are far from Fermi gases [see for instance Eq.(19)]. Later, Lai [55] studied the CFS instability of

\footnotetext{
${ }^{9}$ We remind that polar modes are those whose parity for a given spherical harmonic number $l$ is $(-1)^{l}$ while axial modes have $(-1)^{l+1}$ for parity.
}

composition gravity modes using EOSs that include the strong interaction (see 56]), but in Newtonian rotating NSs. While Yoshida \& Lee [57] looked at the $r$-modes of relativistic stars with gravity modes, yet they mainly focussed on modes due to temperature gradients, and only dealt in a very approximative way with modes due to composition gradients. Going farther in temperature, gravity modes due to discontinuity of density in warm nonrotating relativistic NSs were investigated by Miniutti et al. [58], whereas even more recently gravity modes due to temperature and composition gradients in hot rotating relativistic NSs has been analysed by Ferrari et al. [59], restricting themselves to polar modes. On the other hand, the situation of gravity modes in very cold (superfluid) NSs was first studied by Lee [60], who realised that there was no gravity modes in models with two fluids. This was later confirmed by Andersson \& Comer [61] and Prix \& Rieutord 62], and is now considered as a crucial feature of superfluidity to prove its existence in NSs through the observation of gravitational wave signals.

Nevertheless, as already explained, we shall deal here only with modes of not too cold neither too hot NSs, and the main characteristics of the relevant composition gravity modes resulting from the previously mentioned studies are

(i) a weak coupling to the crust and the independence with respect to the transition radius between the core and the inner-crust $([52], 55])$. Following this result, we decided that it was appropriate to neglect the coupling between the core gravity modes and the crust modes, just giving a boundary condition for the modes at the transition density. Notice that in Paper I we found that using either free surface boundary condition or rigid crust boundary condition was not a key issue in the linear approach to inertial modes. Hence, in all the following, only modes with the boundary condition of a rigid crust (null radial velocity) at a given density are calculated. This density is of course the chosen density for the cutoff of the PAL EOS (see Section IID), i.e. it is either half the saturation density or almost two thirds of it. The influence of the cutoff on the mode shall be discussed in the next subsection;

(ii) the growth time of their gravity driven instability, which is much longer than the time of viscous damping, makes the $g$-modes irrelevant for the production of gravitational waves (at least for cold NSs, see [59]). This comes from their weak coupling to the gravitational field. This consideration is pointless for a nonrotating star, but it explains why in the next section we shall only deal with the current quadrupole that makes $m=2$ inertial modes unstable;

(iii) their frequencies, which are roughly between 50 and 
$400 \mathrm{~Hz}$ for a $M=1.4 M_{\odot} \operatorname{star}([52],[55],[59])$, decrease for a slightly decreasing size of the core.

The latter point turns out to be easy to understand from the usual dispersion law for $g$-modes that can be reached in the Newtonian case using the WKB approach (see the Appendix)

$$
w^{2} \sim \frac{l(l+1)}{(k r)^{2}+l(l+1)} \mathcal{N}^{2},
$$

where $w$ is the frequency, $l$ the quantum number of the decomposition in spherical harmonics, $r$ the radial coordinate, $k$ the radial component of the wave vector and $\mathcal{N}^{2}$ the Brunt-Väisälä frequency defined as

$$
\mathcal{N}^{2} \widehat{=} \frac{1}{n P}|\nabla P|^{2}\left(\gamma_{\beta}^{-1}-\gamma_{F}^{-1}\right) .
$$

Notice that these $\gamma$ are the Newtonian equivalent of the relativistic gamma coefficients ${ }^{10}$ introduced in Section IV and also that in the Appendix this Newtonian formula is written with the sound velocities which are easily related to those coefficients.

Looking at Equation (64), we see that for a given $l$, to make the radius of the star a little smaller (which means to increase the value of $k$ without changing $\mathcal{N}$ ) implies a decrease of $w$ in accordance with point (iii) above.

Since $\mathcal{N}$ depends on the background star, the variation of the frequencies for changes of mass or of EOS are more subtle. To have an insight on this in our case, it is useful to look at the relativistic equivalent of Eq. (65)

$$
\mathcal{N}^{2} \widehat{=} \frac{c^{2}}{P(\rho+P)}|\nabla P|^{2}\left(\gamma_{\beta}^{-1}-\gamma_{F}^{-1}\right),
$$

where we restored explicitly the velocity of light $c$. The values of this frequency $N$ for our models are depicted in Figure 1] whereas Fig 2 shows, as an indication, the gamma coefficients for the models with maximal central densities [A(4) and B(3)]. In Fig 1] we see that the BruntVäisälä frequency (that is an upper-bound for the allowed frequencies) seems to be higher for softer EOSs [comparison between models $\mathrm{A}(4)$ and $\mathrm{B}(3)$ that have the same mass], and increases with the mass for a given stiffness. In the previous studies, this point did not seem very clear, since Reisenegger \& Goldreich found that the frequency of gravity modes increases with mass (which is consistent with the increase of the Brunt-Väisälä frequency), but the result of Lai with fixed mass was that an apparently softer EOS (smaller radius) leads to a smaller BruntVäisälä frequency. Yet, it is worth pointing out that

10 The difference between relativistic and Newtonian gamma coefficients is that the baryonic density $n_{b}$ has to be replaced with the mass density $n$ in the Newtonian case.
Brunt-Väisälä frequency for all used models
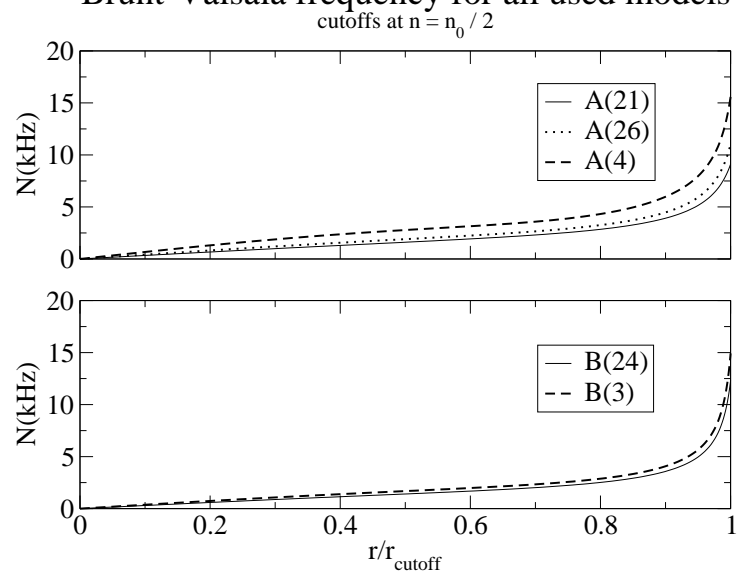

FIG. 1: Brunt-Väisälä frequency for all the models used in this work up to the radius for which the density is half the saturation density. Note that since the frequency vanishes at the center of the star (null gradient of pressure) and monotonously increases with the radius, gravity modes will have an allowed propagation zone with a lower limit.

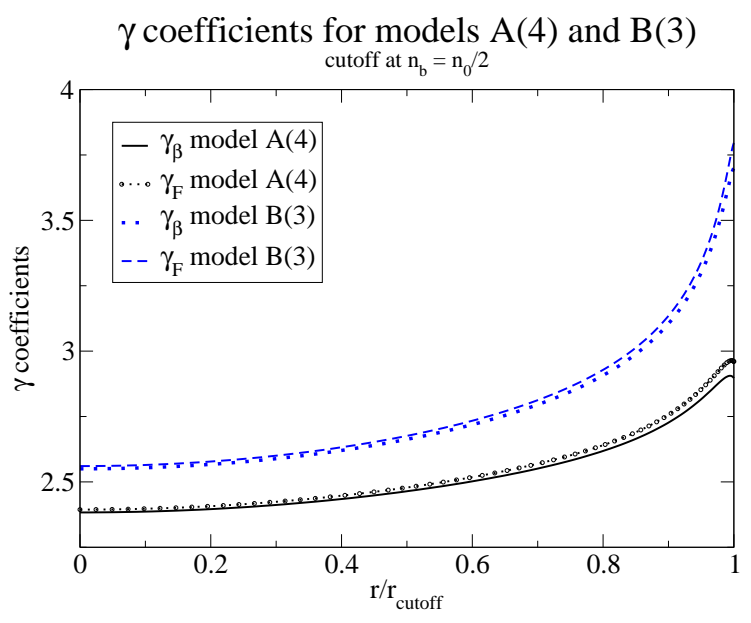

FIG. 2: For the EOSs A and B, $\gamma$ coefficients as functions of the radius (in units of the cutoff radii) in the stars with maximal central densities in order to scan the wider ranges as possible. Notice that the frozen coefficients are always higher than the coefficients at equilibrium, which is a necessary condition for stability.

the properties of a NS depend not only on the compression modulus, but also on the symmetry energy. Since Lai used two completely different EOSs, his results are probably not an indication of the influence of the stiffness on the Brunt-Väisälä frequency nor on the gravity mode frequency. This issue will be addressed in the next section. 
Spectrum for the Lagrangian perturbation of density

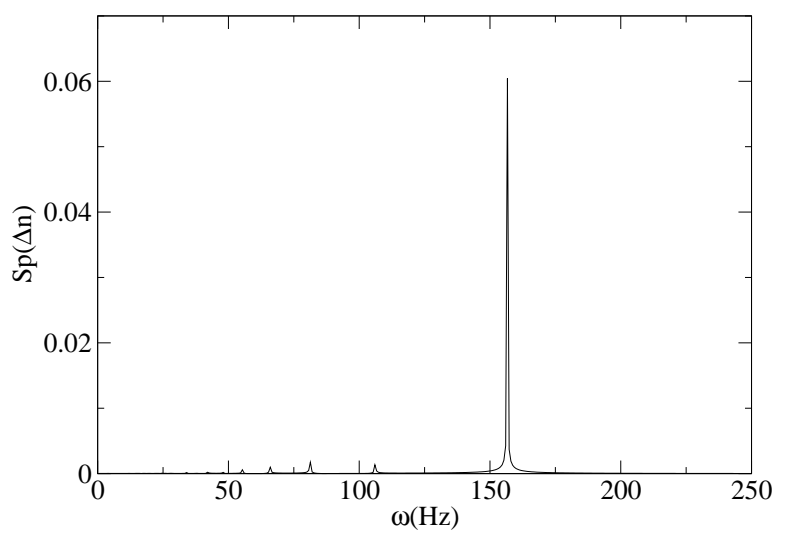

FIG. 3: Fourier power spectrum of the time evolution of a Lagrangian perturbation of density on the equator for $m=2$ initial data. The total physical duration is $1.5 \mathrm{~s}$. See also Figure 4 for a logarithmic representation.

\section{B. Test of the code and gravity modes}

The first test that we did was to take one of the background stars presented in Section 1 D [model A(21)] and perturb it with a small $m=2$ Lagrangian perturbation of density

$$
\begin{aligned}
\Delta n_{b}= & r^{3}\left(1-r^{2}\right) \sin (\vartheta)^{2} \cos (\vartheta) \\
& \times[\cos (2 \phi)+\sin (2 \phi)] .
\end{aligned}
$$

The result and the stability of the code are illustrated in Figures 3 and 4 that depict the Fourier spectra of the evolution. Figure 4 is in logarithmic scale as will be every similar figure in the following: in this way more modes are visible. With this model, the higher frequency gravity mode has a frequency around $150 \mathrm{~Hz}$. This order of magnitude is in agreement with previous results $([52], 55])$, but we shall discuss their values and how they depend on the background star in the following.

In Figure 4 we also put another calculation done with the same background star, but changing the initial data. Instead of using initial data whose angular decomposition corresponds to one single azimuthal number $(m=2)$, we tried initial data with $m=0$ and $m=2$ mixed:

$$
\begin{aligned}
\Delta n_{b}= & r^{3}\left(1-r^{2}\right) \sin (\vartheta)^{2} \cos (\vartheta) \\
& \times[\cos (2 \phi)+\sin (2 \phi)-1] .
\end{aligned}
$$

It can be shown that while the first data were only composed of the $(l=3, m= \pm 2)$ associated Legendre polynomials, the second data additionally contain $(l=1, m=0)$. Hence, this figure shows that, as expected in a spherical star (nonrotating and without anisotropic physics), the modes that can be seen do not depend on the azimuthal numbers present in the angular decomposition. Exception done of the fact that for a given value of $m$ only modes with $l>|m|$ can exist. This is the reason why for $(m=0)$ an additional mode with the frequency $\sim 88.8(\mathrm{~Hz})$ (plus others with very small frequencies) appears.

Since our main goal is to study inertial modes with $m=2$ (see Section VI) that are the most interesting from the gravitational waves point of view, we shall restrict ourselves only to $m=2$ initial data as soon as we shall deal with rotating stars. But for the time being, we shall keep the mixed initial data in the study of gravity modes without rotation. The next point that we shall discuss is the influence of the cutoff density. Following the already mentioned works on gravity modes in NSs, we expect that this cutoff should hardly have an influence on the spectrum, the main influence being linked to the change in the size of the core. This point is clarified by Figure 5 that shows, for the same background star as before, spectra corresponding to the time evolution of the Lagrangian perturbation of (baryonic) density, but also of the radial and $\vartheta$ components of the (Eulerian perturbation of) velocity for two cutoff values: 0.08 or $0.1 \mathrm{fm}^{-3}$. Note that these spectra are reached with evolutions done at different places inside the star. With Figure 5] we are able to verify that to cut at a higher density (which means to make the star smaller) indeed decreases the frequency, but the change is quite small: some $3 \%$ in the worst case. Hence, we shall now restrict ourselves only to calculations done with a cutoff value of $0.1 \mathrm{fm}^{-3}$. Moreover, we also see in this figure that there is a very good agreement between spectra reached from time evolutions of density or of a component of the velocity. In addition, the fact that not all gravity modes have a radial velocity (gravity modes can be either polar or axial) is illustrated, mainly with low-frequency modes.

The next step in our calculation was to look for the influence on gravity modes of the physical parameters that describe the background star. This is depicted in Figure 6] where we draw the frequency of the gravity modes for all models presented in Table I Thus, the three first graphs depict spectra for a relatively stiff matter (compression modulus of $180 \mathrm{MeV}$ ) with increasing central density (from top to bottom) whereas the last two curves are for an EOS which is stiffer: compression modulus of $240 \mathrm{MeV}$. The first comment to make is that to increase the mass (the central density) for a given EOS shifts the spectra to higher frequencies. This result is in agreement with the increase of Brunt-Väisälä frequency found in Figure 1

Next, comparison between the third and the fifth curves also gives a dependence in agreement with Figure 1] These two curves indeed correspond to stars with the same masses, but with different stiffness. Hence, we verify that stiffer EOSs [model $\mathrm{B}(3)$ ] lead to lower fre- 
Spectra for the Lagrangian perturbation of density $(\mathrm{m}=2$ only $+\mathrm{m}=0$ and 2$)$

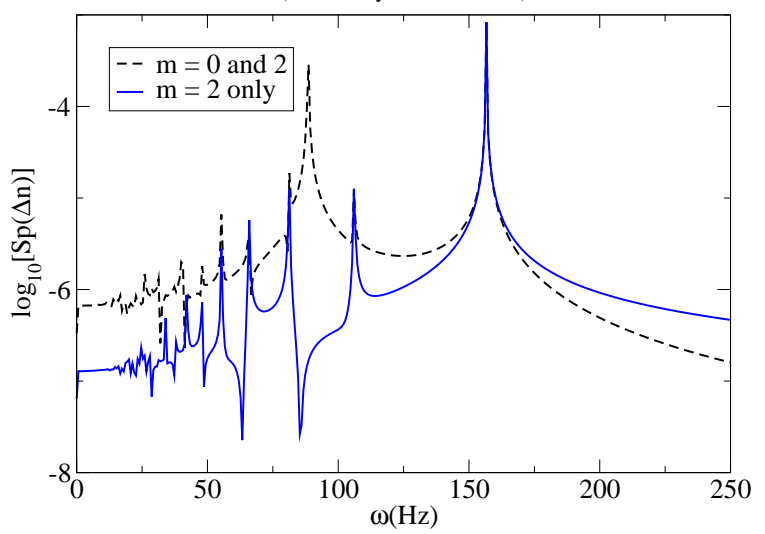

FIG. 4: Same Fourier power spectrum as in Figure 3 but in logarithmic scale. In addition is pictured the spectrum for another calculation that corresponds to a mixture of $m=0$ and $m=2$ as initial data. The agreement concerning the values of the frequencies of the modes is very good and a $l=1$ mode, which only exists for $m=0$, is the main difference between the two spectra.

quencies, even if they imply smaller stars. The radius rather than the mass seems to be the dominant parameter to determine the value of the highest gravity mode. This is made more evident when we look at the second and the third curves [models $\mathrm{A}(26)$ and $\mathrm{A}(4)$ ] and at the fourth and the fifth curves [models $\mathrm{B}(24)$ and $\mathrm{B}(3)$ ]. Indeed, the difference between the mass of $\mathrm{A}(26)$ and $\mathrm{A}(4)$ is about 0.25 solar masses for a difference in the frequency of the highest gravity mode of $17 \mathrm{~Hz}$ (160 to $177 \mathrm{~Hz})$, while for $\mathrm{B}(24)$ and $\mathrm{B}(3)$, the difference between the masses is around 0.2 solar masses with only $5 \mathrm{~Hz}$ between the frequencies $(126$ to $131 \mathrm{~Hz})$. However, as we can see in Table प the difference between the radii is of $0.4 \mathrm{~km}$ for model A and only $0.03 \mathrm{~km}$ for model B. In fact, this result only illustrates the same as in Figure 1 matter added to a star with a stiff EOS hardly changes the radius. This is the reason why the Brunt-Väisälä frequency was changed between models $\mathrm{A}(26)$ and $\mathrm{A}(4)$, but was hardly modified between $\mathrm{B}(24)$ and $\mathrm{B}(3)$. Yet, as we shall see now, when the star is rotating it is more tricky to identify modes just by looking at a spectrum.

\section{INERTIAL MODES AND COMPOSITION GRAVITY MODES IN ROTATING NEUTRON STARS}

\section{A. Modes in rotating stars}

As explained in the Introduction, modes of rotating relativistic stars have the peculiarity that some of them can be driven unstable by their coupling to gravitational waves. In fact, this CFS $([\underline{6}],[]])$ instability is generic, and only insufficiently high angular velocity of a star or physical phenomena like viscosity can prevent some oscillations of being unstable. Furthermore, Friedman \& Schutz [7] proved that the instability appears when an initially retrograde mode becomes prograde (as seen by an inertial observer). To explain how we should be able to "detect" this occurrence in the present work (in which we only look at spectra with positive frequencies), it is worth to start with a brief summary of the main differences between modes of rotating and nonrotating stars.

The first obvious effect of introducing rotation in the Euler equation for the oscillations of a star is to allow the appearance of modes restored by the Coriolis force (the so-called inertial modes). But rotation also breaks the degeneracy at zero frequency of purely axial modes. This leads to the possible existence of purely axial inertial modes, the so-called $r$-modes, while it also possibly couples together axial and polar modes. Another effect of rotation, on the modes that already exist in the nonrotating star, is the splitting of their frequencies. Thus, frequencies measured in the inertial $w_{i}$ and rotating $w_{r}$ frames are linked by the relation

$$
w_{i}=w_{r}-m \Omega,
$$

where $m$ is the azimuthal number and $\Omega$ the angular velocity of the star (or of the rotating frame). Since we now restrict ourselves to modes with $|m|=2$ (the most interesting for GW), this splitting should appear in our spectra calculated in the inertial frame as the replacement of any mode (with frequency $w_{0}$ in the nonrotating case) by a pair of modes with frequencies $w_{r}+2 \Omega$ and $w_{r}-2 \Omega$, with $w_{r} \sim w_{0}$. Notice that the small difference between $w_{r}$ and $w_{0}$ is linked to the influence of rotation on the structure of the star and on the modes frequencies, a difference which should remain quite small since we keep here only terms linear in $\Omega$ (slow rotation approximation).

For such a pair of frequencies, the mode whose positive frequency decreases with increasing $\Omega$ is obviously the retrograde mode. Hence, in Section [VIC] where we shall have gravity modes in rotating stars, a possibly unstable gravity mode will be detected when in the spectra (for increasing $\Omega$ ) its frequency have reached zero and then started to grow again. But as mentioned in Sections IIID and $\mathrm{V}$ gravity modes of cold NSs are not good candidates for the emission of gravitational waves due to the CFS instability. They are weakly coupled to the gravitational field and we shall then not pay too much attention to their potential instability.

The situation is quite different for inertial modes. Indeed, restored (in the Newtonian limit) by the Coriolis force, they only exist for rotating stars and have frequencies proportional to the star's angular velocity $\Omega$. Thus, 


\section{Spectra with change of cutoff density}
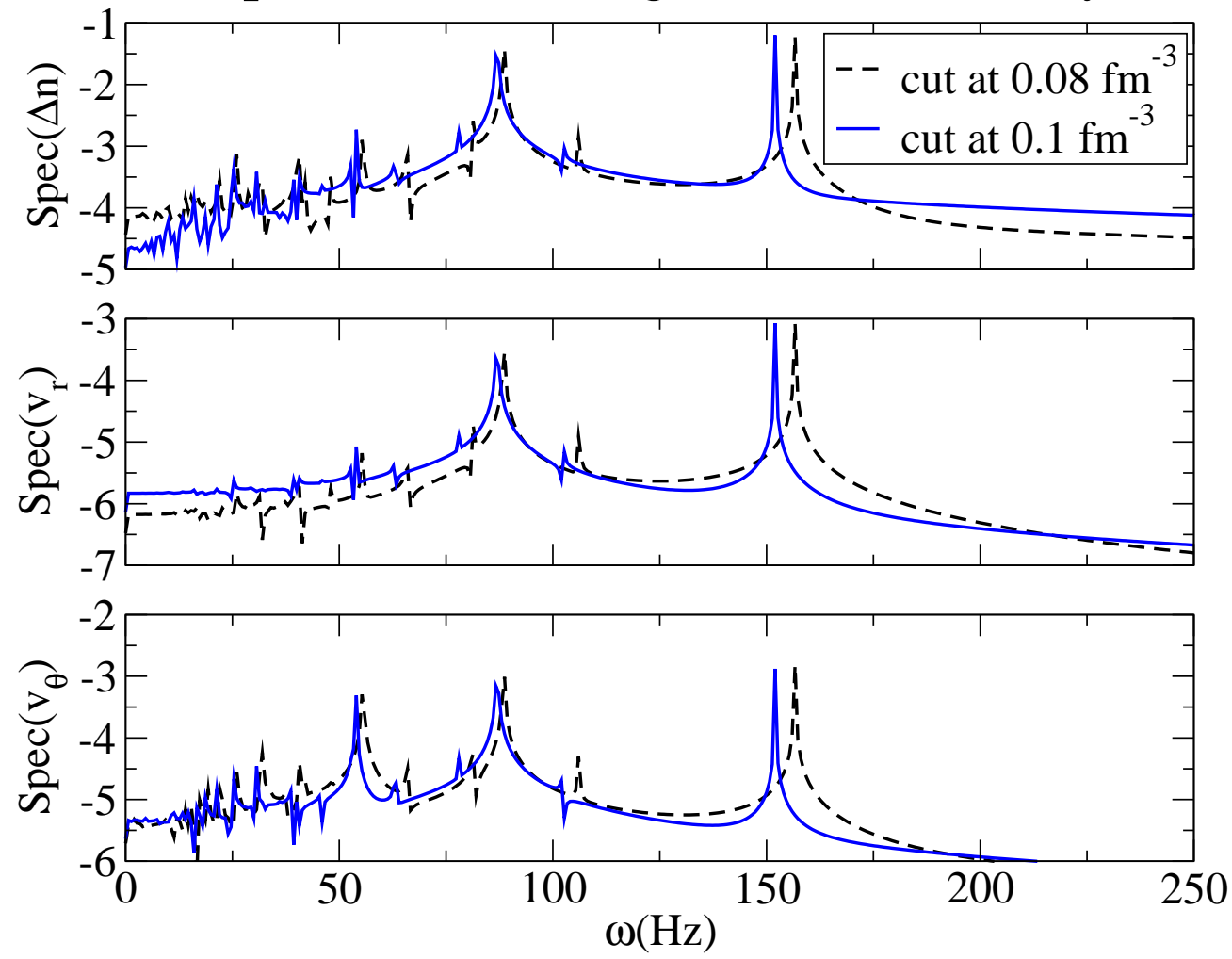

FIG. 5: Fourier power spectra of Lagrangian density perturbation, radial and $\vartheta$ components of velocity for a star with two different values of the density at which is done the cutoff. As expected for gravity modes, the frequencies decrease when the size of the star is made smaller by increasing the value of the cutoff density. Yet, the difference is quite small.

the Newtonian frequency of a purely axial inertial mode is, in the rotating frame,

$$
w_{r}=\frac{2 m \Omega}{l(l+1)}
$$

which gives the always negative frequency (which means an always prograde mode) in the inertial frame

$$
w_{i}=-m \Omega \frac{(l+2)(l-1)}{l(l+1)}=-m\left(\Omega-\frac{2 \Omega}{l(l+1)}\right) \text {. }
$$

This is the reason why, as mentioned in the Introduction, some inertial modes are unstable whatever the angular velocity of the background star is. Moreover, it can be shown ([3], 4], 5]) that depending on the assumed motion (whether or not the perturbations are adiabatic), the properties of inertial modes change. Thus, a Newtonian barotropic star has a spectrum in which only axial modes ( $r$-modes) subsist for spherical harmonics that satisfy $l=m$, whereas nonbarotropic stars admit axial modes for any combination of $l$ and $m$.

In the relativistic case, the situation is a bit different, starting with the fact that no pure axial modes persist for barotropic stars as proven by Lockitch et al. 63] (see also Paper I). For nonbarotropic relativistic stars, the problem was quite controversial during some time and it is still not so clear. Indeed, in the relativistic framework, purely axial modes of nonbarotropic slowly rotating stars are expected to satisfy the Kojima's master equation 64]. This equation contains some perturbations of the metric, but it was shown [5] that the main features of $r$-modes do not change with the relativistic Cowling approximation. Moreover, with this approximation, due to the existence of the frame-dragging effect, this equation easily leads to the relativistic equivalent of Eq. (171)

$$
w_{i}=-m\left(\Omega-\frac{2 \varpi}{l(l+1)}\right)
$$

in which

$$
\varpi \widehat{=} \Omega-N_{\varphi},
$$

where $N_{\varphi}$ is the third component of the shift vector defined in the metric (42). Since $\varpi$ is a function of $r$ (and only of $r$ in the slow rotation approximation), Eq.(72) implies a "continuous spectrum" (see [65] for a proper 


\section{Spectra for gravity modes for different EOSs and masses}

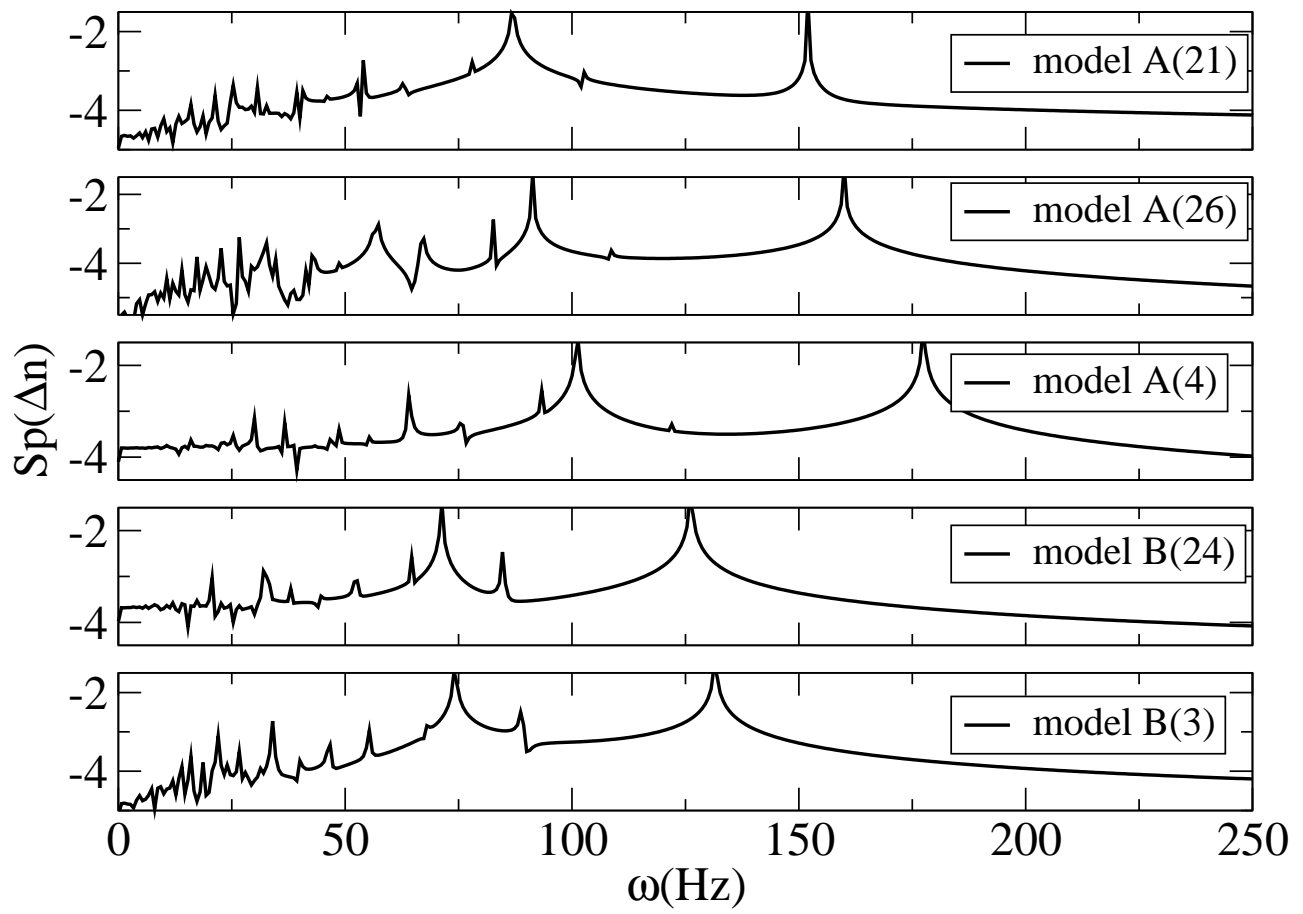

FIG. 6: Fourier power spectra of Lagrangian density perturbation for stars with different masses and EOSs. All the models used in this study are presented here.

demonstration). This made unclear for some time the existence of discrete $r$-modes for certain relativistic stars (for more detail see the review by Kokkotas \& Ruoff 5]). For instance, Ruoff and Kokkotas (see references within 5]) found that only for a very restricted range of polytropic stars was possible to encounter purely axial inertial modes. Thus, the larger is the polytropic index of a star, the lower its maximal compactness can be for it to admit discrete $r$-modes in its spectrum.

But it was also demonstrated 63] that Kojima's master equation becomes a singular eigenvalue problem if the frequency in the rotating frame, $w_{r} \widehat{=} w_{i}+m \Omega$, verifies

$$
\frac{2 m \varpi(0)}{l(l+1)} \leq w_{r} \leq \frac{2 m \varpi(R)}{l(l+1)}
$$

where 0 is the null radius (center of the star) and $R$ the radius at the surface. For this reason, it was claimed by Lockitch \& Andersson [66] that some "boundary layer like" approach could be the way to properly solve this problem. Later Ruoff et al. realized [67] that to include a coupling between $l$ terms of axial modes with $l \pm 1$ terms of polar modes (and respectively) could imply the existence of modes with discrete frequencies, some of them possibly hidden in the continuous part of the spectrum. However, they also found that this continuous spectrum was hugely influenced by the number of $l$ they coupled together.

Thus, the oscillation spectrum of a nonbarotropic rotating relativistic neutron star is still an open problem that we shall deal with in Section VIC in which modes of the background NSs described in Table \will be studied taking into account the frozen composition. Hence, in order to check more easily if the frequencies that will be displayed are inside or outside of the range (74), we summarize, in Table【t their limits for $(m=2, l=2$ or 3$)$ and for all our models ( $c f$. Table I). But since these limits are defined in the rotating frame while we work in the inertial frame, we put in this table not directly $\varpi$ but $\hat{\varpi}_{l m}$ defined as

$$
\hat{\varpi}_{l m} \widehat{=} \frac{1}{\Omega}\left(\frac{2 m \varpi}{l(l+1)}-m\right) .
$$

Finally, to better recognize the influence of the assumption of frozen composition, we shall first focus on inertial modes of those stars with the hypothesis of barotropic EOSs. 
TABLE II: Dimensionless limits of the ranges of continuous spectra of some purely axial inertial modes as seen in the inertial frame for all models presented in Table【 In order to use these limits for arbitrary angular velocity $\Omega$, the dimensionless $\hat{\varpi}_{l m}$ is introduced.

\begin{tabular}{|c|c|c|c|c|c|c|c|c|c|c|}
\hline & & 1) & Moc & & Model & $\mathrm{A}(4)$ & Model & $\mathrm{B}(24)$ & Model & $1 \mathrm{~B}(3)$ \\
\hline$\left(f_{m}-\right.$ & 0.08 & 0.1 & 08 & 0.1 & 0.08 & 0.1 & 0.08 & 0.1 & 0.08 & 0.1 \\
\hline & .602 & & -1.643 & & 731 & & & & & \\
\hline$\hat{\varpi}(0)_{32}$ & -1.801 & & -1.821 & & -1.865 & & -1.816 & & -1.8 & \\
\hline$\hat{\varpi}(R)_{22}$ & 1.422 & & & & -1.468 & & & & -1.459 & -1.455 \\
\hline$\hat{\varpi}(R)_{32}$ & 1.711 & -1.711 & -1.718 & -1.719 & -1.734 & -1.736 & -1.718 & -1.719 & -1.729 & -1.727 \\
\hline
\end{tabular}

\section{B. Modes of relativistic rotating barotropic stars}

The first thing that we shall look at is the influence of our cutoff density on inertial modes. To explore this, we did several time evolutions with the Newtonian $l=m=2 r$-mode as initial data (see Paper I). This purely axial mode was evolved in all backgrounds with different angular velocities of the star, and a typical result is illustrated by Fig 7 This figure depicts the spectra of the $\vartheta$ component of the velocity for a star of model $\mathrm{A}(21)$ with an angular velocity of $50 \mathrm{rad} . \mathrm{s}^{-1}$. Three different values for the cutoff density are used: $0.053,0.08$ or $0.1 \mathrm{fm}^{-3}$, that is to say more or less $1 / 3$, $1 / 2$ or $2 / 3$ of the saturation density $n_{0}$. Moreover, in order to test the possible appearance of a continuous spectrum, we draw the spectra calculated at 2 different positions within the star. Thus, the first obvious result is that for this model at that precise angular velocity, we see a discrete spectrum, with the $l=m=2$ inertial mode (the higher peak in the spectrum) that seems to have a frequency around $73 \mathrm{~Hz}$. This value corresponds to a ratio between the relativistic and Newtonian frequencies in the rotating frame which is around 0.82 , in quite good agreement with the results of Lockitch et al. 68 for that compactness. In addition, various inertial modes can be seen that can be identified as the relativistic counterparts of the inertial modes found by Lockitch \& Friedman [69], but since it was shown 68] that none of these modes are relevant for GW with the CFS instability, we shall not describe them in detail.

Moreover, as can be seen, the influence of the cutoff density on the spectrum of inertial modes is even smaller than for gravity modes. This can probably be understood from the fact that starting with purely axial initial data we reach a radial velocity smaller than in the case of the $g$-modes. This last statement is supported by the comparison between Figures 5 and 8 . Both of these figures depicts spectra for time evolutions of $m=2$ modes in a star of model $\mathrm{A}(21)$. However, for the first one, the spectra of the $\vartheta$ and $r$ components of the velocity correspond to gravity modes in a nonrotating star [initial data identical to the Eq.67) and still without velocity], while for the second they correspond to the Newtonian inertial mode in a barotropic $\mathrm{A}(21)$ star with $\Omega=50$ rad.s ${ }^{-1}$. Notice that for both calculations the cutoff density is $0.1 \mathrm{fm}^{-3}$. What can be seen is that the orders of magnitude of the $\vartheta$ component and of the radial component for the main peak are almost the same for gravity modes (Fig [5], whereas in the case of inertial modes (Fig 8) the radial velocity is more than one order of magnitude smaller than the $\vartheta$ component. This feature can of course also be explained by the fact that the spectra of relativistic inertial modes mainly depend on the compactness of the star as we shall further verify. Notice in addition that, as expected, this inertial mode is not a purely axial mode.

The last curve of Fig 8 is the spectrum of one of the two independent components of the current quadrupole tensor (see Paper I for the exact definition). Since this tensor dominates the post-Newtonian reaction force [51], we shall use it as an indicator of the fastest growing modes, for those that verify the CFS criterion. This indicator tells us that the already mentioned inertial mode actually seems to be the most unstable one. However, even if fewer modes can be seen here than in the spectra of the velocity's components, several other modes appear. Among them, a peak around $47 \mathrm{~Hz}$ but also a quite hidden one around $81 \mathrm{~Hz}$ that is probably the axial-led $l=4$ mode whose stability was discussed by 68]: it has indeed a frequency in the rotating frame that is some $70 \%$ of the frequency of the $l=m=2$ mode, while in the Newtonian case, Lockitch et al. 68] found a ratio of $69 \%$. Notice that this argument relies on the assumption of "almost similarity" of the spectra calculated in the rotating frame for different stars. Formulated in another way, it just means that the spectra in the rotating frames mainly depend on one global physical parameter, the compactness. This assumption will be tested in the following. Anyway, this mode was shown not to be relevant for GW, which is supported by its very low presence (compared with the main peak) in the $S_{i j}$ spectrum. It was more noticeable in the velocity's spectra.

Now that we have analysed the effect of the cutoff density, we shall be able to investigate the inertial modes for all our models. In Fig 9] one can see the same kind of spectra for the $\vartheta$ component of velocity using all our background models and changing the angular velocity from 10 to 80 rad.s ${ }^{-1}$, while in Fig [0] only the curves 


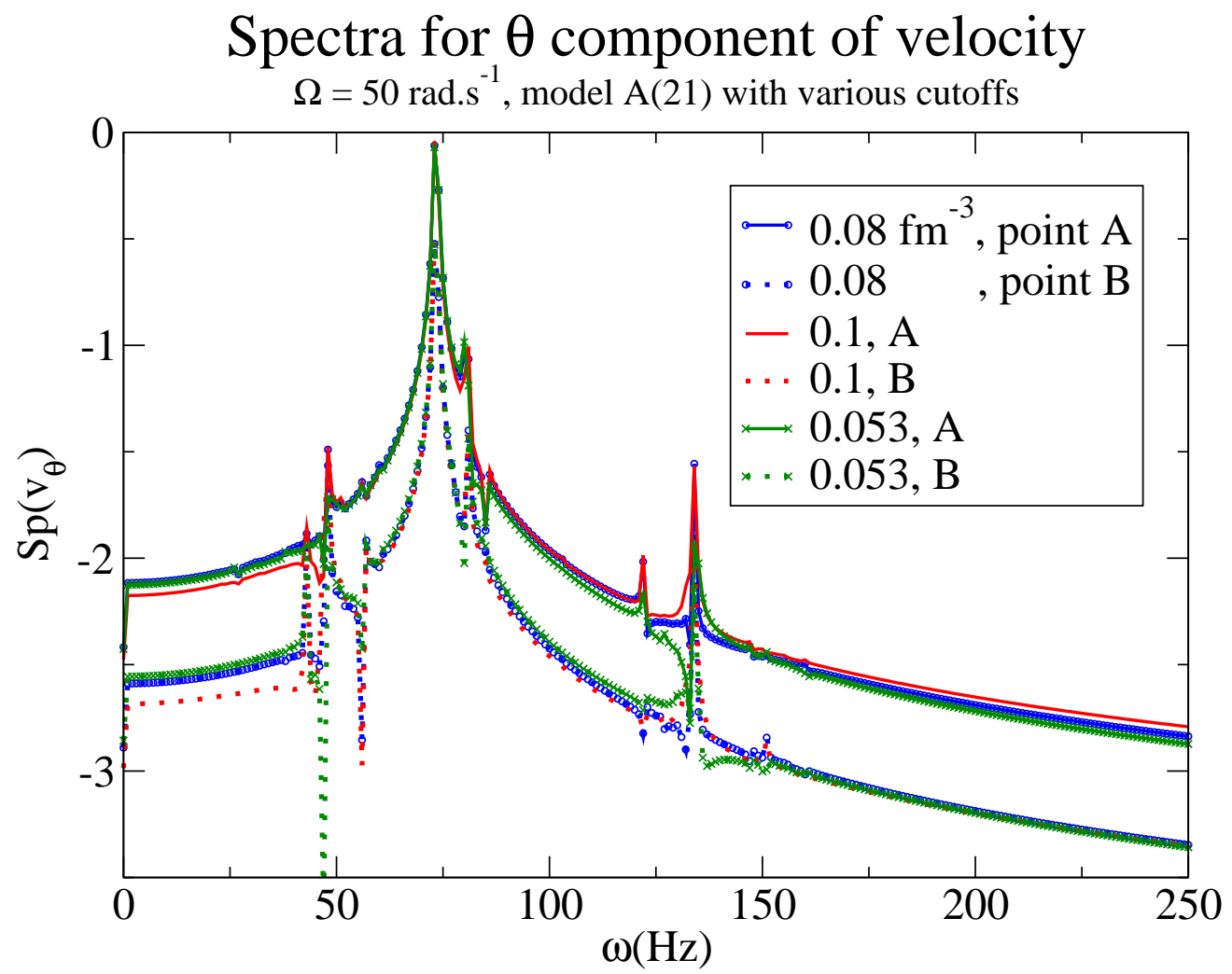

FIG. 7: Fourier power spectra of the $\vartheta$ component of velocity for inertial modes in a barotropic star with various values of the cutoff density. The spectra are calculated at 2 different positions inside the star illustrating the fact that they are discrete modes. Numerous inertial modes can be identified.

Spectra for inertial modes in a barotropic star
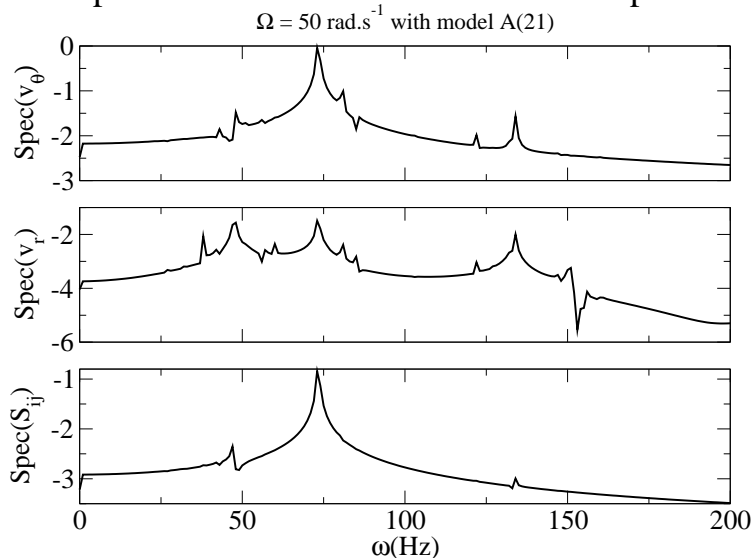

FIG. 8: Fourier power spectra of the $\vartheta$ and $r$ components of velocity and also of one of the 2 independent components of the current quadrupole tensor $S_{i j}$ for inertial modes in a barotropic star with cutoff density $\sim 0.1 \mathrm{fm}^{-3}$. In addition to the $l=m=2$ inertial modes, others can be seen. for $\Omega=80 \mathrm{rad} . \mathrm{s}^{-1}$ are depicted in order to better see the differences between different stars. Thus, the first comment is that the influence of the background star on the spectra is quite small. The higher resolution makes that this is better seen in Fig 10, but already in Fig 9 one can observe a very small dispersion of the frequencies. Moreover looking carefully, one can discern a cross inside the highest square, i.e. the square denoting the $l=2$ inertial mode for model $\mathrm{B}(24)$. Thus, models $\mathrm{A}(26)$ and $\mathrm{B}(24)$ seem to have the same frequency for this mode, which supports the fact that more than the EOS, the compactness of the star plays a key-role in the difference between Newtonian and relativistic frequencies. Indeed, as can be seen in Tab. [1 those stars have almost exactly the same compactness: 0.189 and 0.191 respectively.

Next, to test the dependence with respect to $\Omega$ of the frequencies, we shall divide all of them by the angular velocity of the star. Since it is pointless for gravity modes, we shall restrict this analysis to the $l=2$ inertial mode, whose dimensionless frequency is illustrated versus the angular velocity for all compactness in Fig 11 Nevertheless, before doing more comments about what 


\section{All models for barotropic $\mathrm{m}=2$ inertial modes}

From top to bottom: $\Omega=10,25,50,70$ and 80 rad.s $^{-1}$

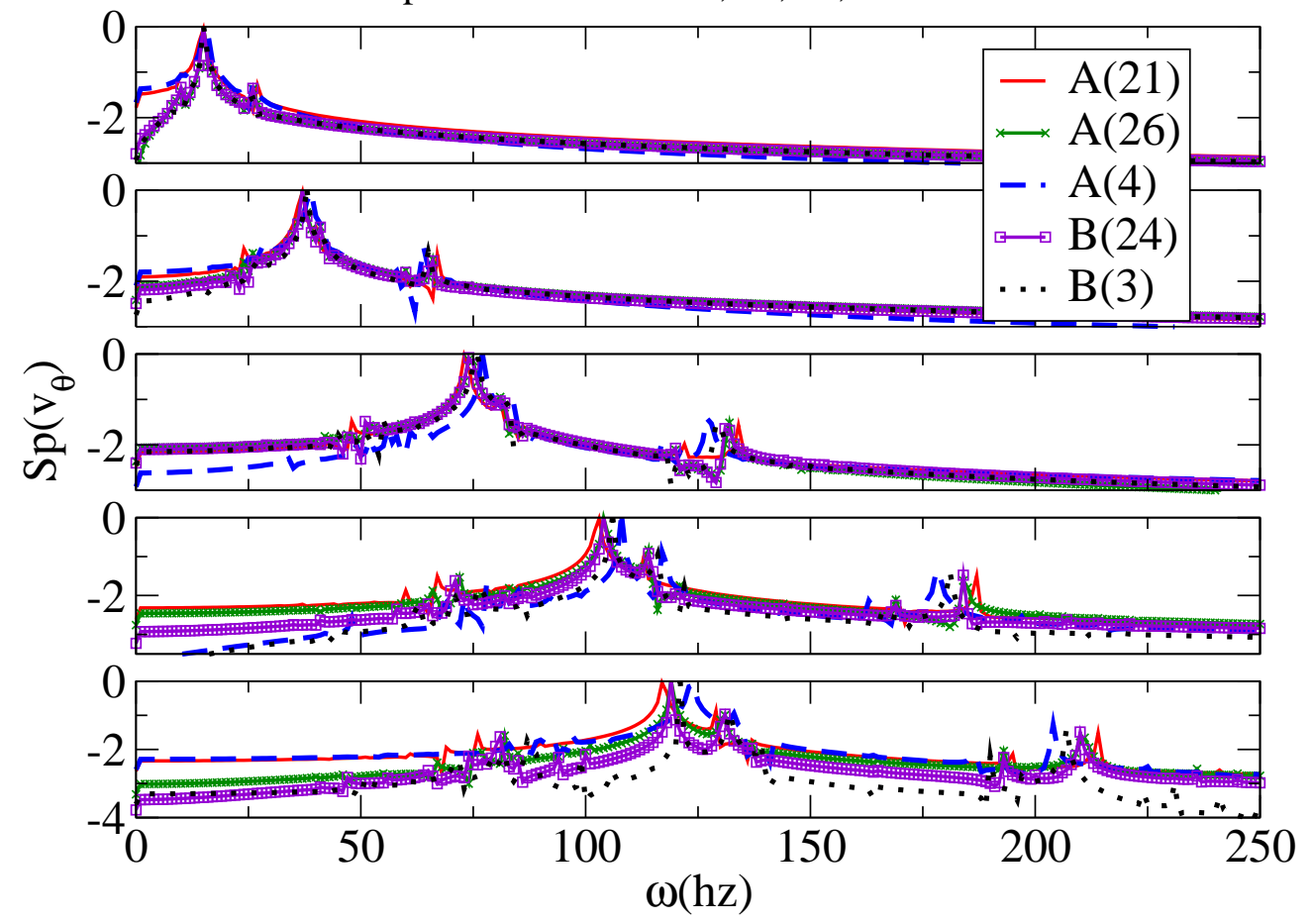

FIG. 9: Fourier power spectra of the $\vartheta$ component of velocity for $m=2$ inertial modes using all our background models with different angular velocity. The star is assumed to be barotropic with a cutoff density at $0.1 \mathrm{fm}^{-3}$.

turns out from this figure, it is worth to stress its limitation. Indeed, the version of the code used in this study works with a given time step of $0.01 \mathrm{~ms}$, and the total duration of the evolutions is always of the order of $1 \mathrm{~s}$, whilst the angular velocity changes. Hence, the error bars on frequencies expressed in $\mathrm{Hz}$ are of about $1 \mathrm{~Hz}$, but for frequencies normalised with the angular velocity $\Omega$, they are equal to $1 \mathrm{rad} . \mathrm{s}^{-1} / \Omega$. Thus, for $\Omega=25$ rad.s ${ }^{-1}$, this is $4 \%$ and this is less than $2 \%$ for $\Omega>50 \mathrm{rad}^{-1} \mathrm{~s}^{-1}$. All of this explains why we did not put the data for $\Omega=10 \mathrm{rad} . \mathrm{s}^{-1}$ that were completely meaningless.

On this other hand, higher angular velocities of the star imply higher frequencies that give better resolution, which support the compactness dependence of the frequency as shown by Fig[11 However, for all angular velocities, the frequencies of the $l=m=2$ mode for models with compactness 0.189 and 0.191 are the same ${ }^{11}$. Yet, our main purpose was not to study inertial modes

11 We verified that for higher frequencies inertial modes, the higher resolution enables us to make a distinction between them. We of barotropic stars, but to try to have an idea of the influence of the microphysical conditions, which implies nonbarotropicity, on the spectra. This is the reason why instead of working with time step and duration scaled by the inverse of $\Omega$ (as we did in Paper I), we use here only "physical scales" that we shall also keep in the next section for the study of the modes of stratified rotating NSs.

\section{Modes of relativistic rotating nonbarotropic stars}

\section{Time evolution of a density perturbation}

The first mentioned effect of rotation was the splitting of noninertial modes. Our investigation of the pulsations of rotating stratified relativistic NSs will begin with the illustration of this phenomenon within the study

shall not discuss this since those modes are not relevant for gravitational waves emission. 


\title{
Spectra for the $\theta$ component of velocity
}

\author{
barotropic $\mathrm{m}=2$ inertial modes for different models with $\Omega=80 \mathrm{rad}_{\mathrm{s}} \mathrm{s}^{-1}$
}

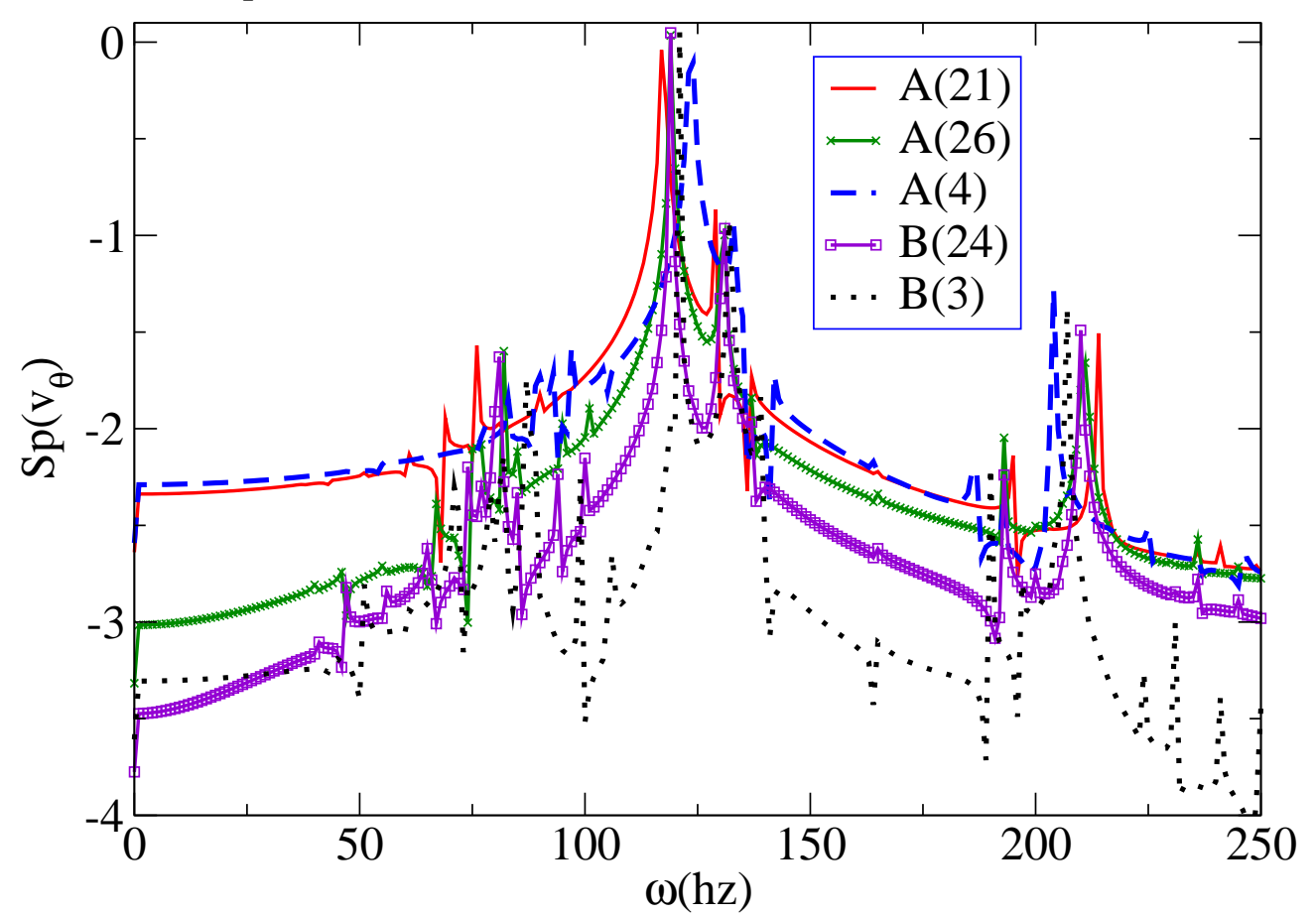

FIG. 10: Fourier power spectra of the $\vartheta$ component of velocity for $m=2$ inertial modes using all our background models for $\Omega=80 \mathrm{rad} . \mathrm{s}^{-1}$. The star is assumed to be barotropic with a cutoff density at $0.1 \mathrm{fm}^{-3}$.

of density perturbations in rotating stratified NSs. To simplify, we shall first restrict ourselves to the model $\mathrm{A}(21)$ with various angular velocities, but keeping the cutoff density at $0.1 \mathrm{fm}^{-3}$. In fact, since we have verified in the previous sections its low influence, we shall use in the following only that value of the cutoff density.

Fig 12 depicts the spectra of the Lagrangian perturbation of density. The initial data consist of the same $m=2$ perturbation of density [see Eq. (67)] without any velocity as in Section $\mathrm{VB}$ As expected, the splitting of the $g$-modes can be seen, but in addition, we can observe the emergence of some probable inertial modes: see the peaks around $120 \mathrm{~Hz}$ and $150 \mathrm{~Hz}$ for $\Omega=80 \mathrm{rad} . \mathrm{s}^{-1}$. The main reasons why these modes can be thought to be inertial modes are that both their frequencies and their relative importance in the spectra grow with the angular velocity. Yet, to better describe them, it is worth to have a look at the spectra of the velocity in Figs 13 and 14 where we show spectra of the $\vartheta$ and radial components of the velocity, respectively.

First of all, Fig 13 directly shows that not all modes were visible in the spectra of the density. Thus, in addition to the splitted gravity modes and to the already mentioned candidates inertial modes, there are many others that do not correspond to peaks in the density spectra. We shall now see how we are lead to classify them as axial-led inertial modes, following the classification of Lockitch et al. 63. Obviously, they are inertial modes since their frequencies increase with the angular velocity. But our view of classifying them as axial-led is supported by Fig 14 which shows that the radial velocity for those modes is very small comparatively to the radial velocity of others. Moreover, one of them even seems not to have any radial counterpart (at this scale of precision): the mode with a frequency of $116 \mathrm{~Hz}$ for $\Omega=80$ rad.s ${ }^{-1}$, that is to say the mode with the lowest frequency among those axial-led inertial modes.

Interestingly, Fig 15] which depicts the spectra for the current quadrupole tensor, shows that this mode is the mode with the largest contribution to this tensor. Notice anyway that its amplitude is very low (logarithmic scale) due to the initial data. Nevertheless, for all the preceding reasons, we can now say that this is the $l=m=2$ inertial mode, and that Table III tells us that its frequency is inside the range of the continuous 


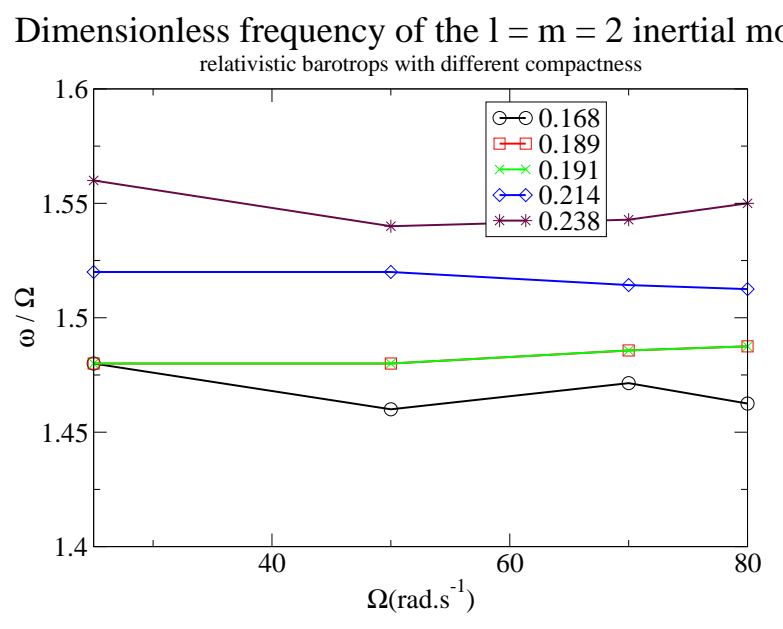

FIG. 11: Dimensionless frequency versus angular velocity of the star for $l=m=2$ inertial mode in barotropic stars of various compactness. Notice that due to the fact that evolutions lasted $1 \mathrm{~s}$, the error bar is equal to $1 \mathrm{rad} . \mathrm{s}^{-1} / \Omega$.

spectrum. Indeed, its dimensionless frequency in the inertial frame is 1.45 whereas for model $\mathrm{A}(21)$ the range is $[1.42,1.6]$. Moreover, with the already displayed spectra of velocity, the spectra of a global quantity (the current quadrupole), plus various spectra of velocity calculated in several positions within the $\operatorname{star}^{12}$, we verified that this is a discrete eigenvalue.

Finally, before we try to investigate the $l=m=2$ inertial mode in more detail by changing the initial data, let us introduce some axial and polar "kinetic energies" that shall help us to study the coupling between polar and axial parts of the modes. What we shall call "kinetic energy" in the following was already introduced in Paper $\mathrm{I}$ and is defined as

$$
\hat{E} \widehat{=} \frac{1}{2} \int_{\text {Star }} \mathrm{d}^{3} \mathrm{~V} \tilde{\mathrm{n}} \vec{W}^{2},
$$

where $\tilde{n} \hat{=} n_{b} N[r]^{2} a[r]$ [see Eq. (48)], $\vec{W}$ is the three dimensional vector field (the Eulerian perturbation of velocity) defined in Eq. (46) and $\mathrm{d}^{3} \mathrm{~V}$ is the flat elementary volume.

With the anelastic approximation, this quantity can be shown to be conserved for a barotropic EOS and a vanishing density at the surface, conditions that were verified in Paper I. But for nonbarotropic EOSs with a cutoff density, two ways to violate this conservation exist. The first one, linked to the EOS, corresponds to

12 We do not show the corresponding graphs here, but they are very similar to what was done in Fig 7 for the barotropic case. the existence of a "chemical" part of the physical energy, while the second is linked to fluxes through the surface. To calibrate the violation of the conservation due to fluxes, we did time evolutions of this kinetic energy in barotropic stars with cutoff densities $0.053,0.08$ or 0.1 baryon. $\mathrm{fm}^{-3}$. We found that for these values, the higher was the cutoff, the better was the conservation, which means that our usual cutoff at 0.1 baryon. $\mathrm{fm}^{-3}$ is the best of them. Notice that in all cases we saw temporary violations of the conservation, but the temporal mean value of the energy was always conserved. Moreover, even in the worst cases, the violation were never higher than $0.6 \%$. On the other hand, testing the influence of the "chemical energy", we verified that for nonbarotropic EOSs with a cutoff at 0.1 baryon. $\mathrm{fm}^{-3}$, the mean value was no longer conserved, but the violation was only of the order of some 3 percents. For all these reasons, we shall work in the following with this (kinetic) energy as a not too bad indicator of the energetic behavior of the oscillations, at least as far as the coupling between polar and axial parts is concerned.

Indeed, we remind that our algorithm for the solution of Euler's equations (see Paper I) relies on the use of the radial velocity and two scalar potentials whose angular divergence and curl enable us to recover the usual spherical components. By using these variables, the separation of the polar and axial parts of the velocity field is done very easily, which naturally leads to the polar and axial kinetic energies. Thus, Fig [16 that displays the time evolution of the ratio between the polar kinetic energy and the total kinetic energy give a first insight on the energy flows between the polar and axial parts of the modes.

In this figure, time evolutions of the ratio are shown, whose duration is only $200 \mathrm{~ms}$ in order to make visible the "quasistationnarity" that is reached after a while. As can be seen, starting with a purely polar perturbation, the proportion of the energy stored in polar modes is indeed decreasing down to a fraction that is between 80 and $90 \%$, depending on the angular velocity of the star: the faster it rotates, the more energy flows to axial modes. Notice anyway that since the initial data are a perturbation of density without any velocity, at the very beginning, there is, rigorously speaking, no kinetic energy. But in fact, from the very first time steps the perturbation of density implies a velocity which is initially purely polar. Moreover, we encountered quite similar results when we did some tests with as initial data a purely polar velocity field without any density perturbation. Only the ratio was slightly changed, whilst the time scales were identical. Nevertheless, the relevant issue concerning energy flows will be how barotropicity affects this evolution. This will be one of the topics of the next section. 


\section{Model $\mathrm{A}(21), \mathrm{m}=2$, cutoff $=0.1 \mathrm{fm}^{-3}$}

From top to bottom: $\Omega=10,25,50,70$ and 80 rad.s $^{-1}$

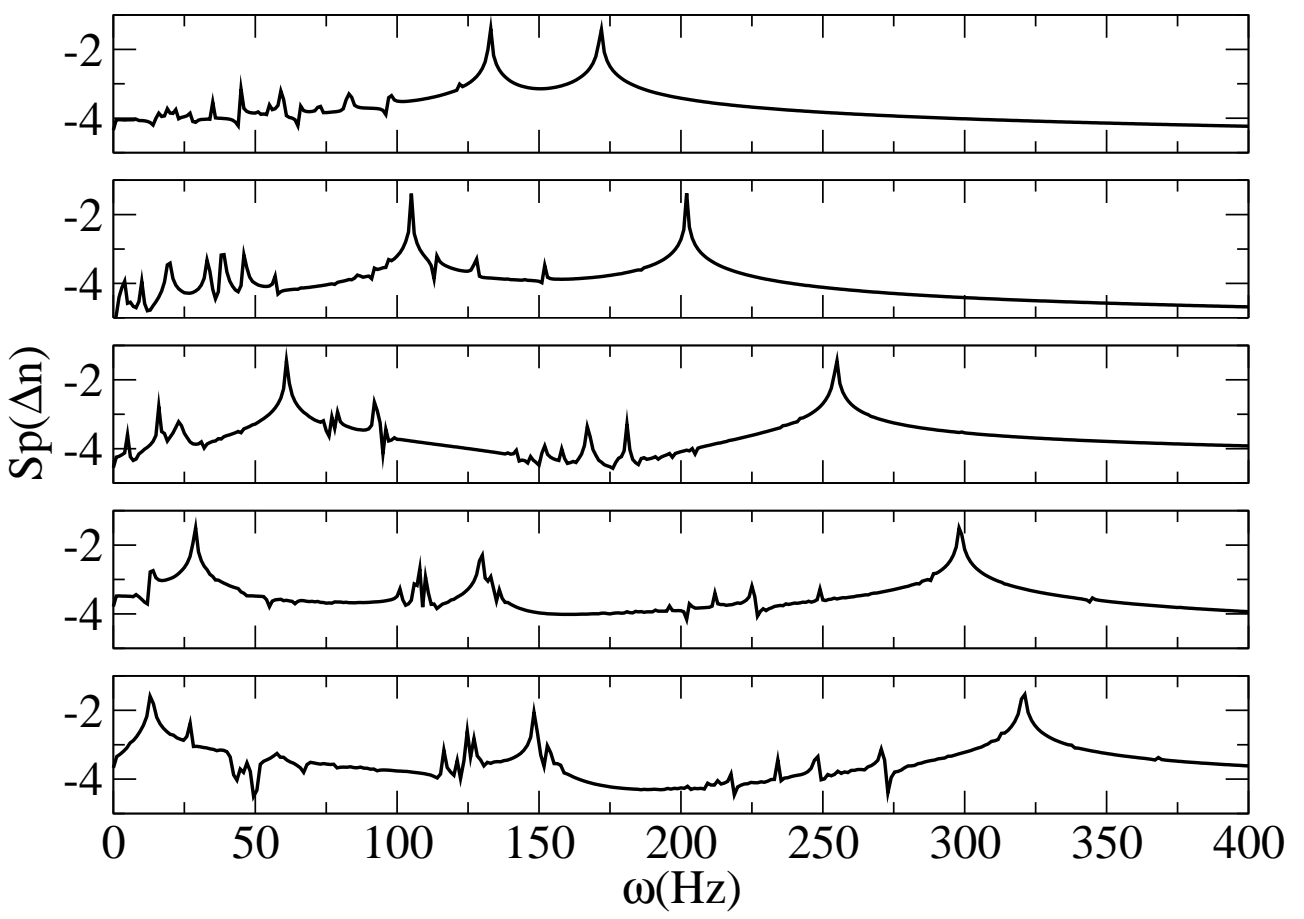

FIG. 12: Spectra of the Lagrangian perturbation of density for several angular velocities. The star is nonbarotropic and of model A(21), while the initial data are a $m=2$ density perturbation. See also Figs. 13] 14] and 15]

\section{Time evolution of the $l=m=2$ inertial mode}

We shall now study the time evolution of oscillating out-of-equilibrium NSs with different initial data: the $l=m=2$ inertial mode instead of a density perturbation. In this way, the differences with the barotropic case shall be easier to notice, while we should also be able to see how much our previous results for nonbarotropic stars depend on the initial data. To start with, Fig [17 depicts the spectra of the Lagrangian perturbation of density for this case, with the results already presented in Fig 12 for comparison. The first thing to comment on is that even for low angular velocity, gravity modes appear, with, as expected, an amplitude that grows with $\Omega$. But it shall also be pointed out that the $l=2$ and $l=4$ inertial modes are visible, even if we consider the density perturbation spectra, and that their amplitudes stay the same for all values of $\Omega$. But the coupling between axial and polar parts of the velocity field should be better investigated with the spectra of the velocity components. These are displayed in Figs 18 and 19

Fig 18 shows that the $\vartheta$ component of the velocity is hardly affected by the fact that the star is a barotrop.
The main difference between the spectra is indeed only that, for nonbarotropic stars, gravity modes appear, with amplitudes that become higher for faster rotating stars. Even the frequency of the $l=m=2$ inertial mode is almost exactly the same. But on the other hand, the radial part of the velocity (Fig [19) is completely changed and quite strongly depends on the angular velocity, which was predictable. Thus, for slowly rotating stars, gravity modes dominate and the radial velocity of the inertial mode is some two orders of magnitude lower than in the case of a barotropic star. Yet, for increasing angular velocity, the amplitude and the frequency of the radial part of the $l=m=2$ inertial mode become more and more similar to what they are in a barotropic star. For $\Omega=80{\mathrm{rad} . \mathrm{s}^{-1}}^{-1}$, there is less than one order of magnitude difference between the barotropic and the nonbarotropic cases for the $l=2$ mode (whose frequency in the inertial frame is $w_{i} \sim 116 \mathrm{~Hz}$ in this case), whereas there was two orders for $\Omega=10 \mathrm{rad} . \mathrm{s}^{-1}$. On the other hand, it should be pointed out that also the amplitude of the $g$-modes grows with $\Omega$ (probably meaning that their "inertial" part is on the way to dominate buoyancy) while many of the inertial modes that existed for barotropic stars have disappeared. 


\section{Model $\mathrm{A}(21), \mathrm{m}=2$, cutoff $=0.1 \mathrm{fm}^{-3}$}

From top to bottom: $\Omega=10,25,50,70$ and 80 rad.s $^{-1}$
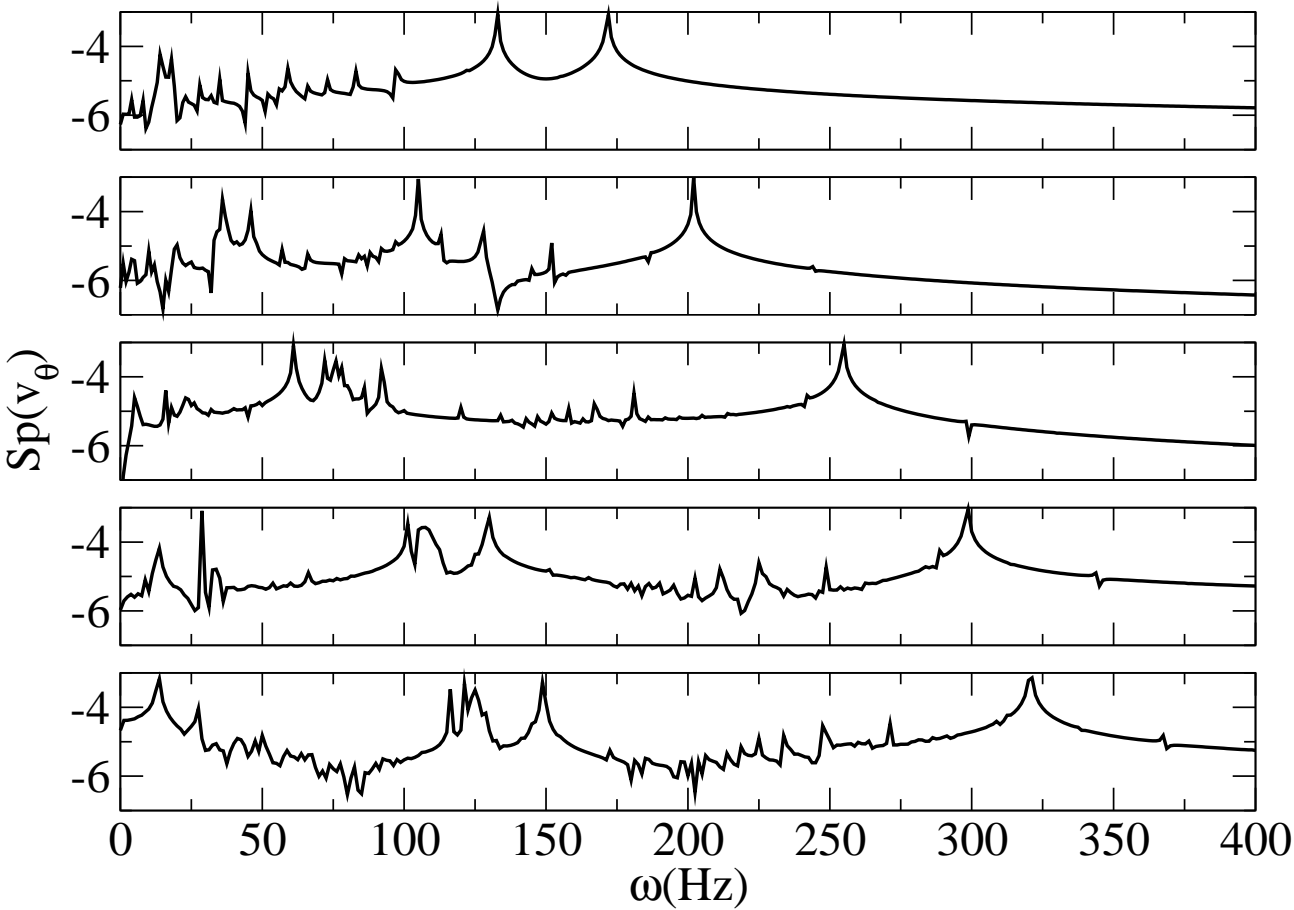

FIG. 13: Spectra of the $\vartheta$ component of velocity for several angular velocities. The star is nonbarotropic and of model A(21), while the initial data are a $m=2$ density perturbation. See also Figs. 12 14 and 15]

If we now look at Fig 20 that depicts the spectra for the current quadrupole tensor, we shall observe that from the point of view of $\mathrm{GW}$ emission, barotropicity seems not to have a major impact, at least in the spectrum. Indeed, the spectra of this tensor do not show remarkable differences, with only a small shift in the frequency of the probably most unstable mode that can be pointed out. We shall now investigate in more detail how this shift in the frequency depends on the EOS, after a brief comment on the time evolution of the ratio between polar and axial kinetic energies.

Indeed, while all previous spectra were very comparable, the time evolutions of the polar and axial parts of the kinetic energy are quite different for barotropic and nonbarotropic stars. The latter situation is illustrated by Fig[21] (out-of-equilibrium case), and by Fig[22 (barotropic case) $)^{13}$. Thus, one observes that, for a

13 Notice that evolutions in the barotropic case are, as expected, analogous to the result presented for a relativistic polytrop in barotropic star, energy is fast flowing back and forth from the axial part to the polar part, leading to a kind of "stationary state" with a medium value of the ratio around $2 \%$. On the other hand, for nonbarotropic stars, the coupling between polar and axial parts seems to depend much more on the angular velocity. Hence, for $\Omega=10$ or 25 rad.s ${ }^{-1}$, even after $600 \mathrm{~ms}$ of evolution, the "final state" is still not reached and the ratio still grows. But the curves for higher angular velocities suggest that they should not get too high since the "stationary situations" obtained in those cases are characterized by ratios lower than for barotropic stars (always less than 1\%).

The difference between the typical times is quite easy to understand. Indeed, energy flows should have for typical time scales the periods of the modes measured in the rotating frame. For nonbarotropic stars, whatever the angular velocity, some $g$-modes exist with periods of some ms, which are then responsible for the quite

Paper I. 


\section{Model $\mathrm{A}(21), \mathrm{m}=2$, cutoff $=0.1 \mathrm{fm}^{-3}$}

From top to bottom: $\Omega=10,25,50,70$ and 80 rad.s $^{-1}$

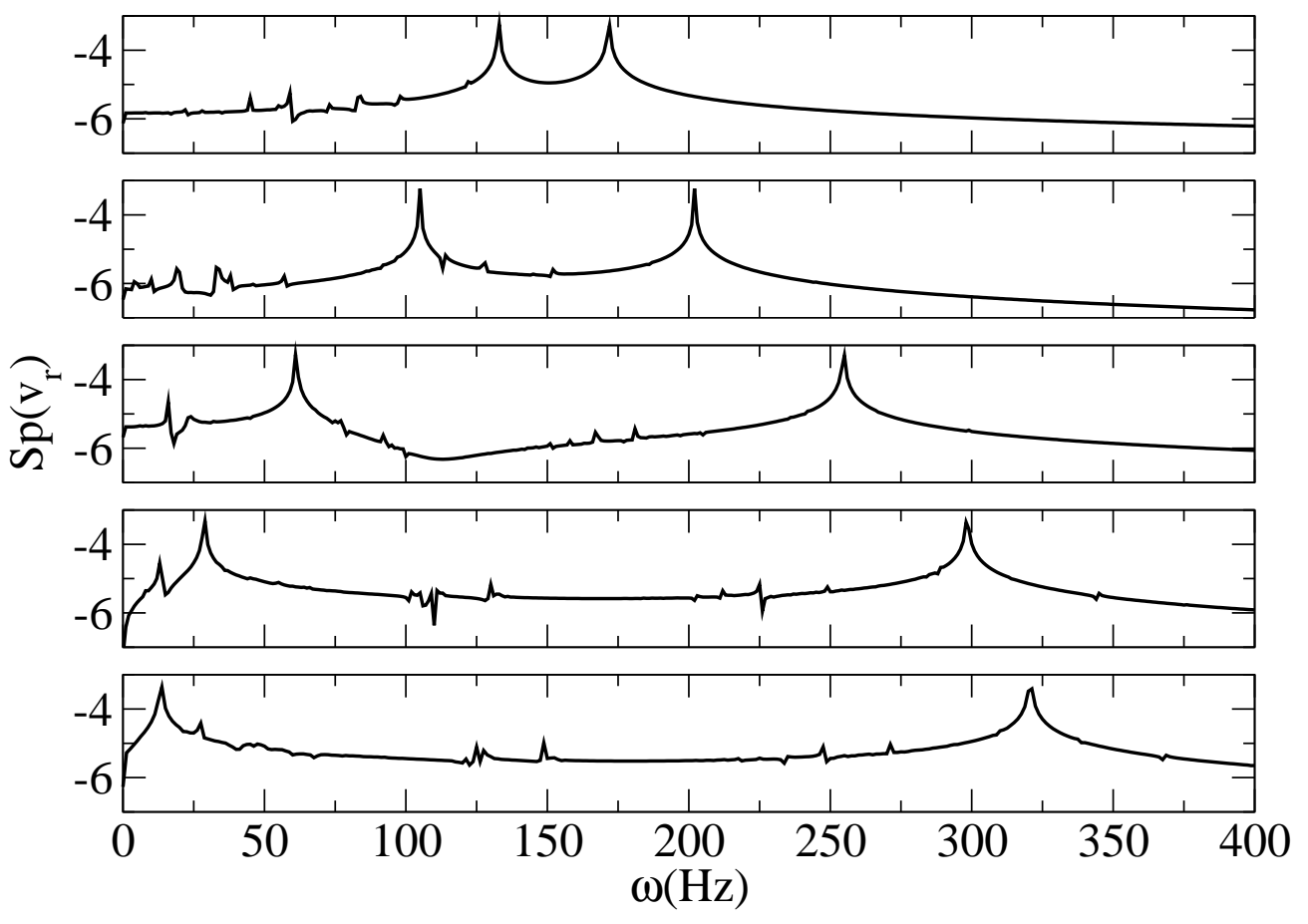

FIG. 14: Spectra of the radial component of velocity for several angular velocities. The star is nonbarotropic and of model $\mathrm{A}(21)$, while the initial data are a $m=2$ density perturbation. See also Figs. 12] 13] and [15]

fast flows. However, in the barotropic case, only inertial modes can be observed. Since they have periods proportional to the inverse of the angular velocity, this is the explanation of the two features of the barotropic case: the self-similarity (for different values of $\Omega$ ) of the time evolutions and the acceleration of the flows with $\Omega$.

On the other hand, with some caution due to our numerous approximations (and also to our definition of "kinetic energy"), we could say that the result concerning amplitude of the ratios can be seen as an indication than stratification probably helps the unstable inertial mode (the $l=m=2 r$-mode) to be driven to instability by $\mathrm{GW}$ since its coupling to polar modes (which are not driven to instability by the current quadrupole) is smaller. Yet, the answer to that question requires a proper treatment of the emission of gravitational waves without the Cowling approximation, while different viscosities for polar and axial modes should also complicate the problem.

To conclude this work, we shall give an overview of how the frequency of the $l=m=2$ inertial mode depends on compactness, barotropicity and angular velocity. The latter will be the easier: as already discussed, due to the error bars for the normalized frequencies, we are not really able to see any difference. Moreover, since our study is linear in $\Omega$, we expect that the normalized frequency of the inertial modes should only depend on the compactness of the stars but not on $\Omega$. This view is supported in the barotropic case and in the nonbarotropic case by (respectively) both Figs 11 and 23. Thus, the more compact a NS is, the higher will be its dimensionless frequency as measured by an inertial observer. However, the comparison between these two figures shows that taking into account the fact that npe matter should keep a frozen composition, which implies a nonbarotropic EOS, leads to lower frequencies. Moreover, it is worth pointing out that we verified that, for all of our models, the $l=m=2$ inertial modes had discrete spectra $^{14}$, even if Fig 23 and Tab. III demonstrate that all these modes were found within the range of the continuous spectra. Nevertheless, as already mentioned and as can be seen

\footnotetext{
14 which was easier to see by looking at the current quadrupole tensor that is a global quantity.
} 


\section{Model $\mathrm{A}(21), \mathrm{m}=2$, cutoff $=0.1 \mathrm{fm}^{-3}$}

From top to bottom: $\Omega=10,25,50,70$ and 80 rad.s $^{-1}$

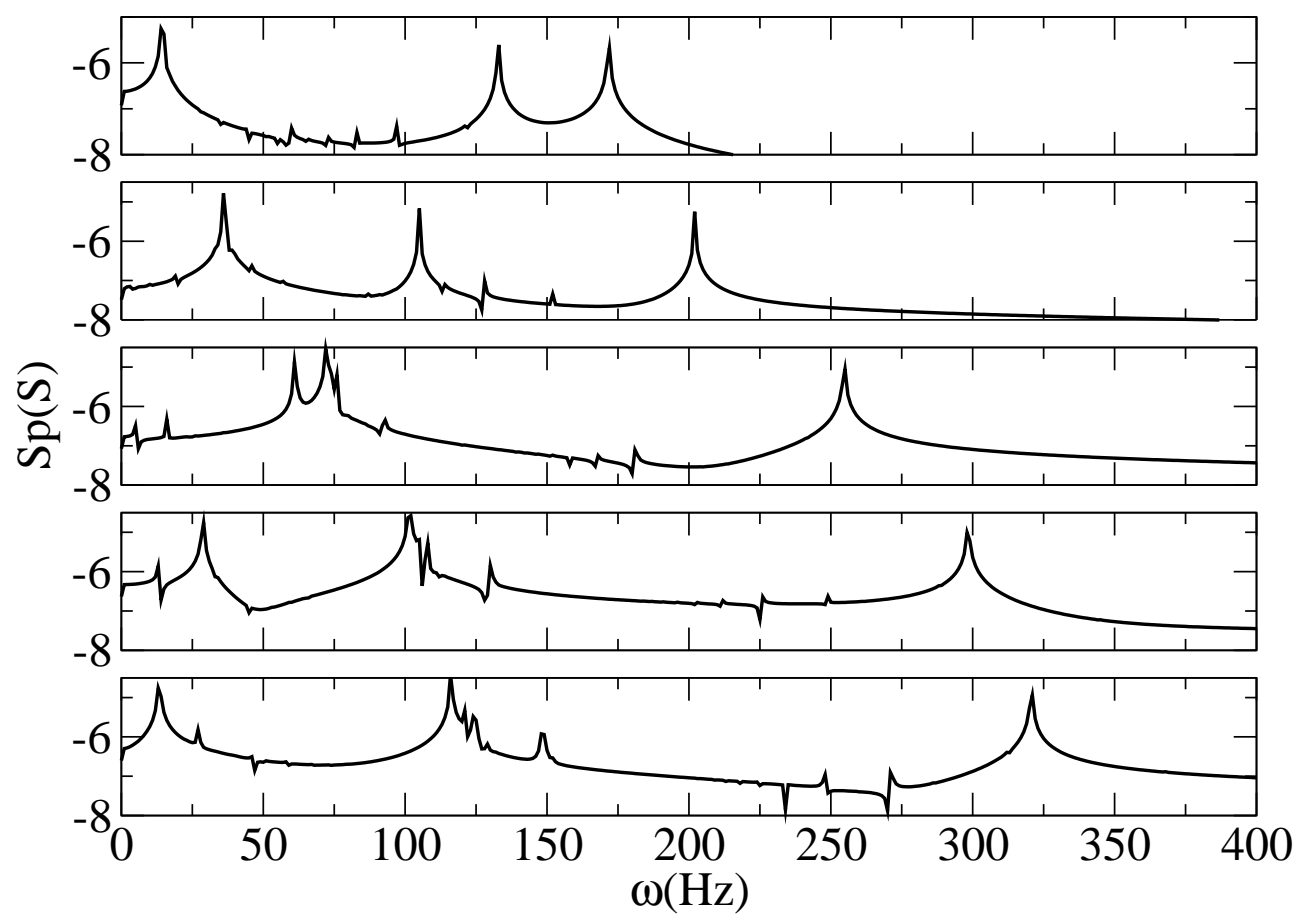

FIG. 15: Spectra of the current quadrupole tensor $S$ for several angular velocities. The star is nonbarotropic and of model $\mathrm{A}(21)$, while the initial data are a $m=2$ density perturbation. See also Figs. 12] 13] and 14.

in Fig [19] they are modes with a radial velocity, which means that they are not purely axial. For very slowly rotating stars, this radial component is quite small, but it becomes as large as what it is for nonpurely axial inertial modes of barotropic stars when the angular velocity increases. Finally, notice that even if models $\mathrm{A}(26)$ and $\mathrm{B}(24)$ have the same compactness and then the same frequency for the $l=m=2$ inertial mode, due to the mixing with gravity modes that depends on the detail of the microphysics, their full spectra are quite different. This issue is illustrated by Fig 24 that depicts the spectra of the radial component of the velocity for those models with the out-of-equilibrium hypothesis. Hence, this probably means that more precise studies including more coupling between modes (nonlinear terms in $\Omega$ or nonlinear corrections to the Euler equations) should enable us to make some differences between stars of the same compactness, and then to start to probe the inner composition of NSs when gravitational waves emitted by their instabilities are detected.

\section{CONCLUSION}

Inertial modes and gravity modes of slowly rotating relativistic stratified neutron stars have been investigated in the linear regime. Our study is based on a spectral three dimensional evolutionary code which has, at the moment of writing, the restrictions of using the Cowling and the anelastic approximations. However, the effect of the latter approximation is mainly to kill high frequency acoustic modes that should not be strongly coupled to inertial and gravity modes for slow rotation. Additionally, the slow rotation approximation is reasonable for observed pulsars as, even for the fastest known pulsar, the pertinent dimensionless parameter is of the order of some percents. The validity of the Cowling approximation is more subtle since without perturbations of space-time we do not directly include gravitational waves in our calculations, whereas they are one of the main motivations for this work. Nevertheless, previous studies have proven that the properties of inertial modes were not too much affected by its use [5]

Another major feature of our work is that we tried to simulate as well as possible the microphysical conditions 


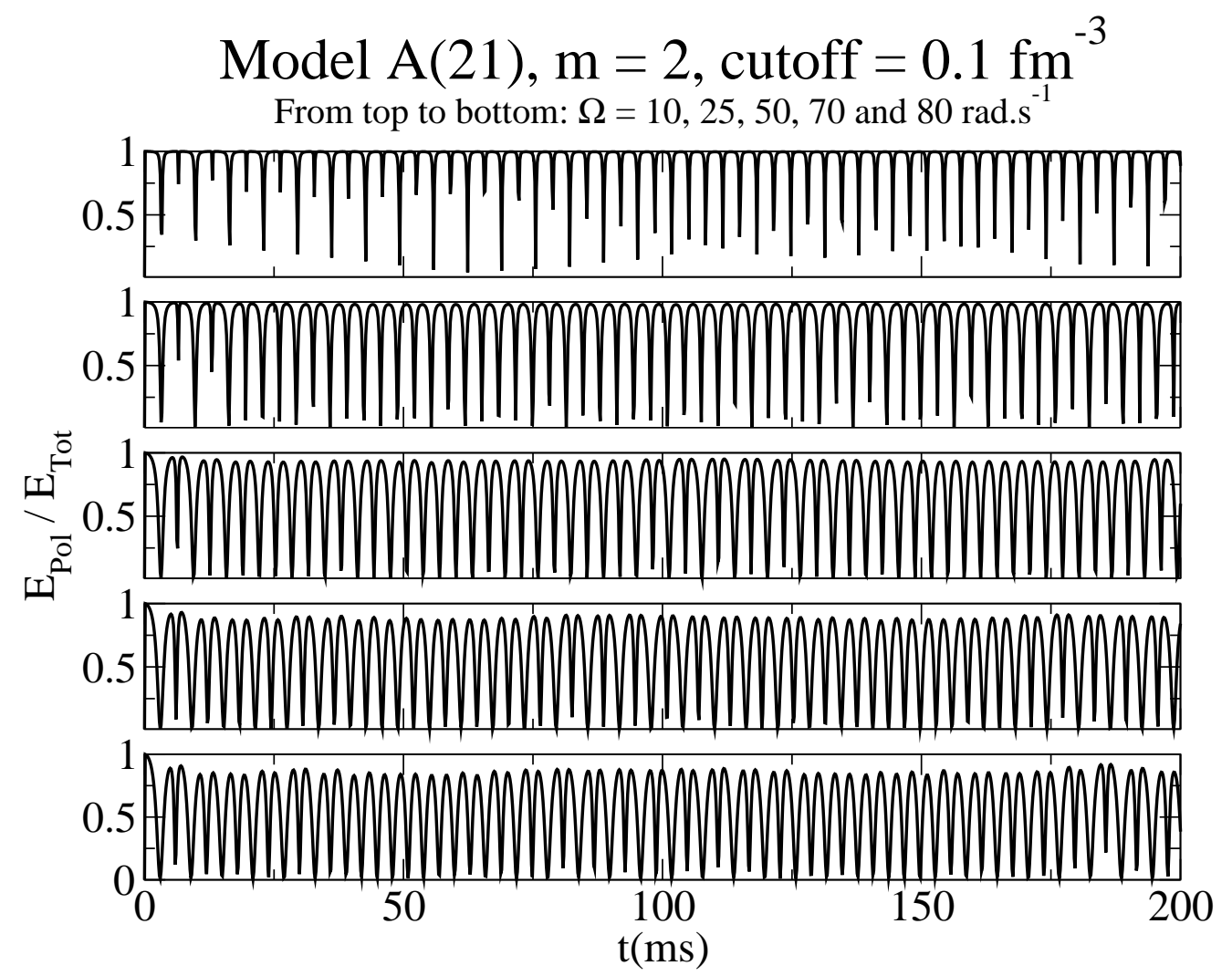

FIG. 16: Time evolution of the ratio between the polar kinetic energy and the total kinetic energy (as measured in the rotating frame) in the same star as in previous figures: a nonbarotropic star of model $\mathrm{A}(21)$.

that should occur inside not too massive and not too cold neutron stars. Thus, by using a quite realistic equation of state for nuclear matter valid even when the beta equilibrium is broken, we did the first three dimensional time evolution of inertial modes in stratified relativistic stars, taking into account the frozen composition of every perturbed lump of npe matter. With this approach, we were able to show that the coupling between polar and axial modes quite strongly increases with the angular velocity. This happens in such a way that, in nonbarotropic stars, initially axial inertial modes develop a radial part that can be as large as the polar part of the general inertial modes of barotropic stars. Yet, several of the polar-lead inertial modes of barotropic stars do no longer exist in nonbarotropic stars. Moreover, we found that the characteristic time scale for the exchanges of energy between polar and axial modes is different for barotropic and nonbarotropic stars. Thus, the coupling of inertial modes with gravity modes in nonbarotropic stars seems to give birth to fast energy interchange between the polar and axial parts of the fluid motion. This phenomenon could have some implications on the way viscosity or gravitational waves emission act, but it would of course need further studies to draw robust conclusions.

However, the comparison of inertial modes in stars with and without the assumption of frozen composition has already lead us to the conclusion that nonbarotropicity decreases the influence of compactness on the frequency of the $r$-mode, making in addition this frequency slightly lower in a nonbarotropic star. If further studies with different equations of state and composition support this result, it could mean that the measurement of the global parameters of a neutron star (compactness, mass and radius if absorptions lines are observed, binary system parameters, etc.) in conjunction with the observation of gravitational waves emitted by some instabilities of the inertial modes would be very instructive about the inner structure of neutron stars. But such a Grail would probably need to first improve our understanding of the physics with wider explorations of the physical parameters: centrifugal terms and deformation of the star, magnetic field, differential rotation, nonlinear coupling, etc. Among them, superfluidity is one of the key phenomena, since several studies proved that gravity modes are suppressed by its existence. As a consequence, even if superfluidity in old neutron stars is already strongly sup- 


\section{Model $\mathrm{A}(21), \mathrm{m}=2$, cutoff $=0.1 \mathrm{fm}^{-3}$}

From top to bottom: $\Omega=10,25,50,70$ and 80 rad.s $^{-1}$

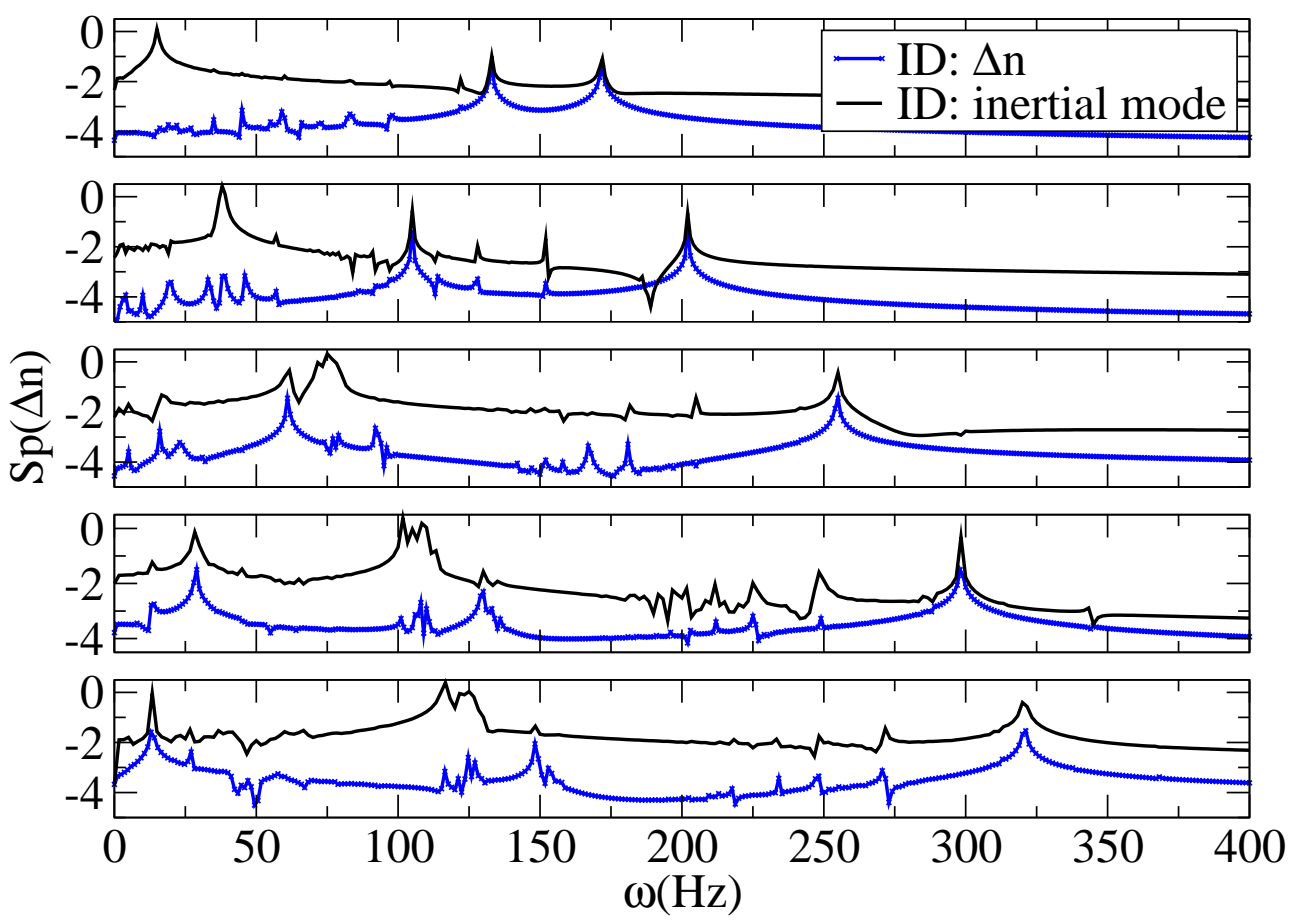

FIG. 17: Spectra of the density perturbation for several angular velocities of the star for an initial $l=m=2$ inertial mode evolving in a nonbarotropic star of model $\mathrm{A}(21)$.

ported by both theory and observation (glitches), the detailed analysis of the gravitational wave spectra of oscillating neutron stars could be the possibility to definitely demonstrate the superfluid nature of the neutron stars content by the lack of well understood features linked with gravity modes.

\section{Acknowledgments}

We are indebted with J.A. Pons for his very critical reading of the manuscript and for his numerous comments, but it is also a real pleasure to thank N. Andersson and A. Nagar for many useful suggestions. Finally, we would like to thank the computer department of the Paris-Meudon Observatory for the technical assistance. This work has been supported by the EU Programme 'Improving the Human Research Potential and the SocioEconomic Knowledge Base' (Research Training Network Contract HPRN-CT-2000-00137). PH was partially supported within the program of European Associated Laboratory "Astrophysics Poland-France" (Astro-PF), and LV benefited from the Jumelage PAN-CNRS Astronomy France-Poland.

\section{APPENDIX A: DISPERSION RELATIONS}

In this Appendix, the usual basic way to derive the Newtonian dispersion relation for $g$-modes is summarized, and we briefly discuss the effect of the divergence free and anelastic approximations.

\section{Usual case with Cowling approximation}

The easiest way to reach the dispersion relation for gravity restored modes is based on the perturbative Lagrangian approach. If we restrict ourselves to linearized equations, and write $\vec{\xi}$ the displacement vector, $\Delta$ the Lagrangian perturbations and $\delta$ the Eulerian (linked by the symbolic relation $\Delta \widehat{=} \delta+\vec{\xi} \cdot \vec{\nabla}$ ), we have for the Euler equation

$$
\partial_{t}^{2} \vec{\xi}=-\frac{1}{n} \vec{\nabla} \delta P-\vec{\nabla} \delta \phi-\frac{\delta n}{n} \vec{\nabla} \phi
$$

and for the mass conservation equation

$$
\Delta n=-n \vec{\nabla} \cdot \vec{\xi},
$$




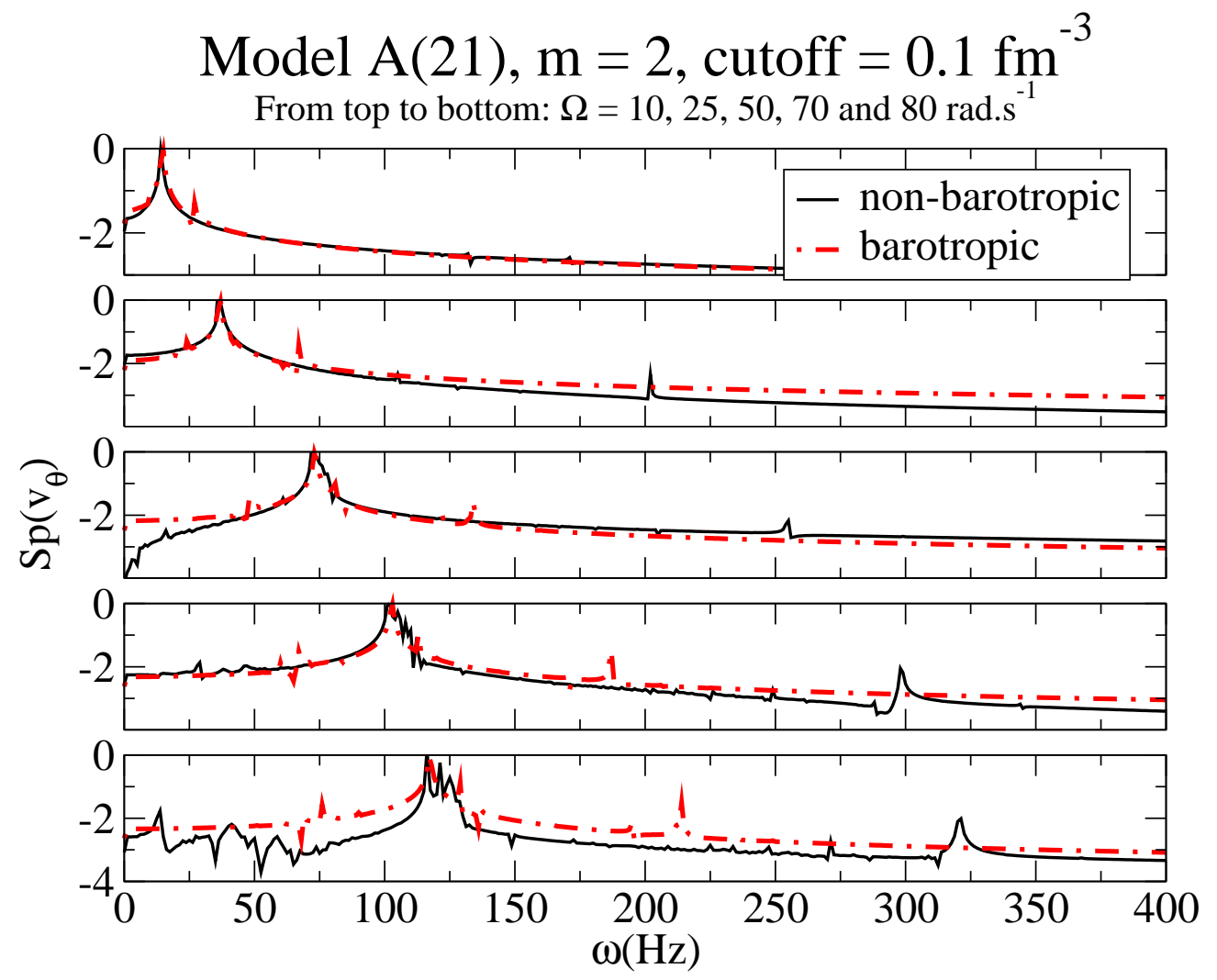

FIG. 18: Spectra of the $\vartheta$ component of velocity for several angular velocities in a barotropic and nonbarotropic star of model $\mathrm{A}(21)$ with inertial mode for initial data.

where $P$ is the pressure, $\phi$ the gravitational potential and - indicates the usual Euclidean scalar product.

If we now consider only spherical nonrotating stars (only radial dependence for $\phi$ ) and neglect the Eulerian perturbation of the gravitational field (Cowling approximation), the nonradial part of Eq.(A1) and Eq.(A2) give us

$$
\Delta n=n\left(\frac{1}{r^{2}} \partial_{r}\left(r^{2} \xi_{r}\right)-\frac{l(l+1)}{r^{2} w^{2}} \frac{\delta P}{n}\right)
$$

in which we assumed a decomposition in spherical harmonics of the scalar variables $[l$ is the index of the spherical harmonic $\left.Y_{l}^{m}(\vartheta, \varphi)\right]$ and a harmonic time dependency of $\xi$ :

$$
\partial_{t}^{2} \vec{\xi} \hat{=}-w^{2} \vec{\xi}
$$

Introducing now the equilibrium $c_{e q}$ and frozen composition (adiabatic) $c_{F}$ speeds of sound,

$$
|\nabla P| \widehat{=} c_{e q}^{2}|\nabla n|
$$

and

$$
\Delta P \widehat{=} c_{F}^{2} \Delta n,
$$

with the Brunt-Väisälä frequency defined as

$$
\mathcal{N}^{2} \widehat{=}\left|\frac{\nabla P}{n}\right|^{2}\left(c_{\beta}^{-2}-c_{F}^{-2}\right),
$$

we obtained the following system of equations

$$
\begin{aligned}
\frac{1}{r^{2}} \partial_{r}\left(r^{2} \xi_{r}\right)+\frac{\delta P}{n c_{F}^{2}}\left(1-{\frac{w_{0}}{w}}^{2}\right)-\frac{g \xi_{r}}{c_{F}^{2}} & =0 \\
\frac{1}{n} \partial_{r} \delta P+\frac{g}{c_{F}^{2}} \frac{\delta P}{n}-\left(\mathcal{N}^{2}-w^{2}\right) \xi_{r} & =0,
\end{aligned}
$$

with the Lamb frequency defined as $w_{0}^{2} \widehat{=} c_{F}^{2} l(l+1) / r^{2}$ with $g$ the local gravitational acceleration.

The new variables

$$
\begin{aligned}
& X \widehat{=} r^{2} \xi_{r} e^{A}, \\
& Y \widehat{=} \delta P e^{-A},
\end{aligned}
$$

with

$$
A \widehat{=}-\int \frac{g}{c_{F}^{2}} \mathrm{dr},
$$

enable to write the system as 


\section{Model $\mathrm{A}(21), \mathrm{m}=2$, cutoff $=0.1 \mathrm{fm}^{-3}$}

From top to bottom: $\Omega=10,25,50,70$ and 80 rad.s $^{-1}$

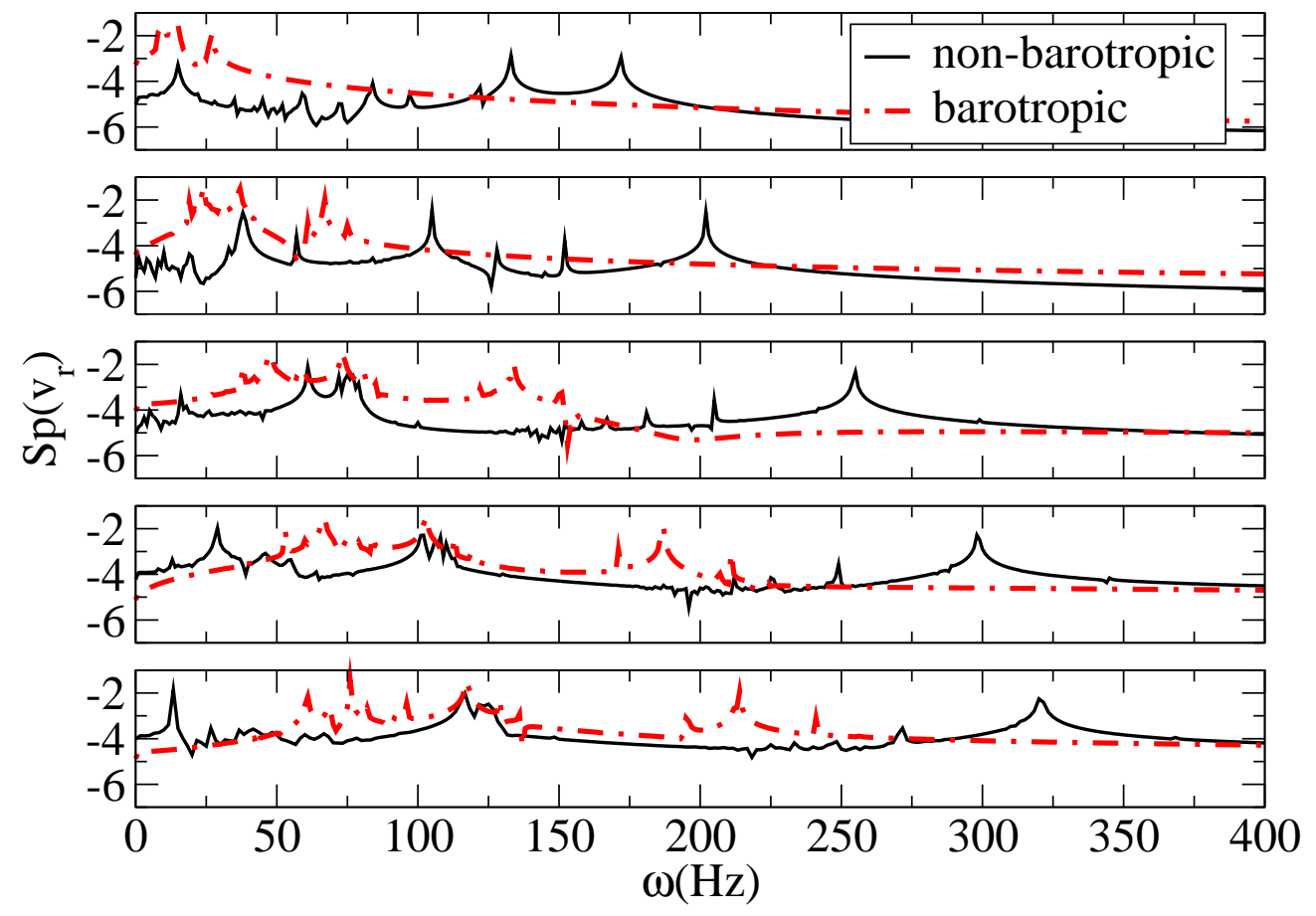

FIG. 19: Spectra of the radial component of velocity for several angular velocities in a barotropic and nonbarotropic star of model $\mathrm{A}(21)$ with inertial mode for initial data.

$$
\partial_{r} V=\left|\begin{array}{cc}
0 & r^{2} e^{2 A}\left(w_{0}^{2} / w^{2}-1\right) /\left(n c_{F}^{2}\right) \\
n e^{-2 A}\left(w^{2}-\mathcal{N}^{2}\right) / r^{2} & 0
\end{array}\right| V,
$$

with

$$
V \hat{=}\left(\begin{array}{c}
X \\
Y
\end{array}\right)
$$

A WKB-like approximation,

$$
\partial_{r} V=i k V
$$

gives the dispersion relation

Eq. (A16) leads to the two asymptotic and separated branches for the $p$-modes

$$
w^{2}={\frac{c_{F}}{r}}^{2}\left((k r)^{2}+l(l+1)\right)
$$

and the $g$-modes

$$
w^{2}=\mathcal{N}^{2} \frac{l(l+1)}{(k r)^{2}+l(l+1)} .
$$

$$
k^{2}=\left(\mathcal{N}^{2}-w^{2}\right) \frac{1}{c_{F}^{2}}\left(\frac{w_{0}^{2}}{w^{2}}-1\right) .
$$

Since for neutron stars we have

$$
\mathcal{N} \ll \frac{g}{c_{F}} \ll c_{F} k,
$$

\section{Divergence free and anelastic approximations}

The two approximations that we shall now discuss differ by the way they treat the continuity equation A2 . The divergence free approximation consists, as indicated 


\section{Model $\mathrm{A}(21), \mathrm{m}=2$, cutoff $=0.1 \mathrm{fm}^{-3}$}

From top to bottom: $\Omega=10,25,50,70$ and 80 rad.s $^{-1}$
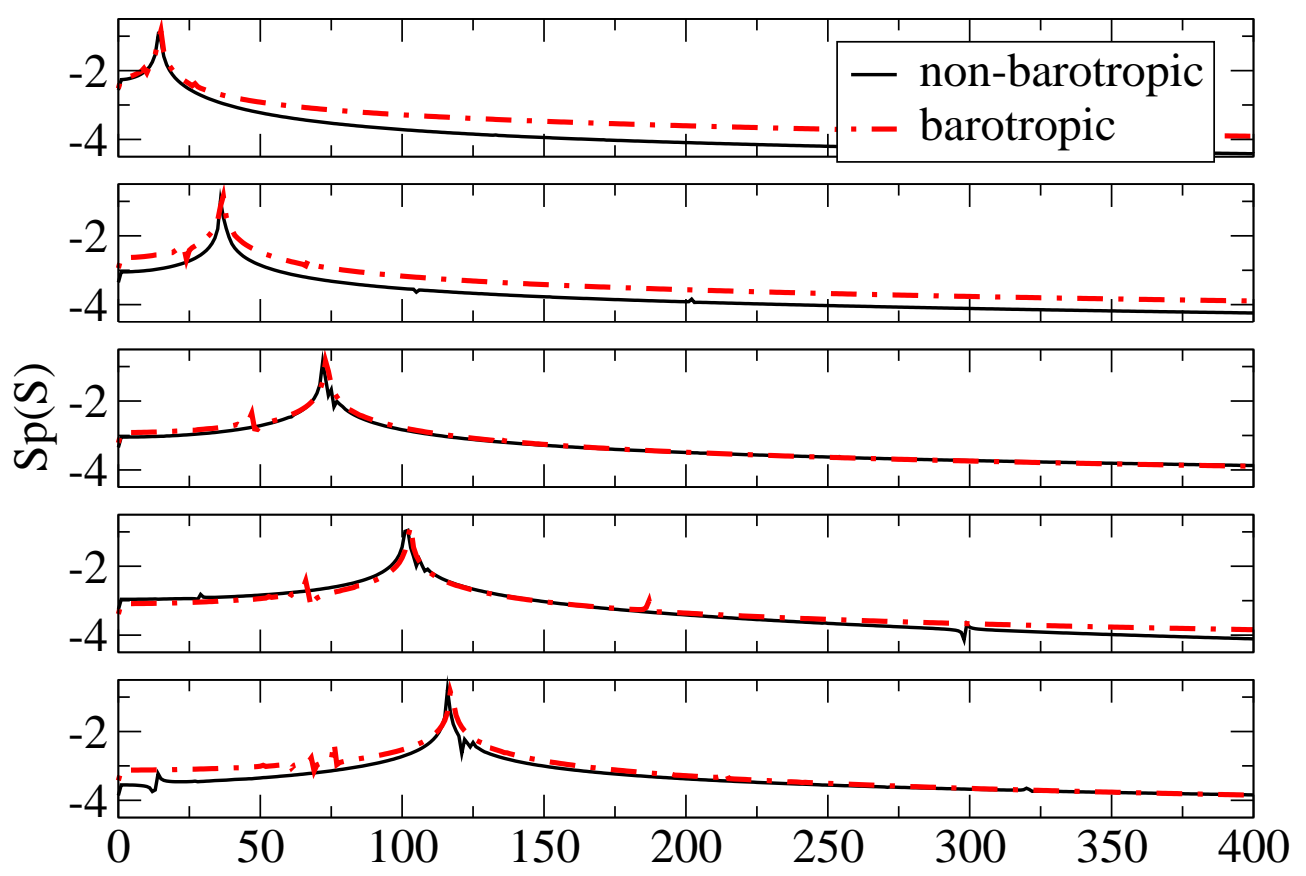

FIG. 20: Spectra of the current quadrupole tensor for several angular velocities in a barotropic and nonbarotropic star of model $\mathrm{A}(21)$ with inertial mode for initial data.

by its name, in the replacement of it by the condition

$$
\vec{\nabla} \cdot \vec{\xi}=0
$$

while the anelastic approximation neglects the time variation of the Eulerian perturbation of density, which gives

$$
\vec{\nabla} \cdot(n \vec{\xi})=0
$$

In the case of the anelastic approximation, some algebra shows that the system A13) is replaced with

$$
\partial_{r} V=\left|\begin{array}{cc}
0 & r^{2} e^{2 A} w_{0}^{2} /\left(n c_{F}^{2} w^{2}\right) \\
n e^{-2 A}\left(w^{2}-\mathcal{N}^{2}\right) / r^{2} & 0
\end{array}\right| V
$$

which leads to the relation (A19) as the unique solution: the $p$-modes have been "killed" by the anelastic approximation.

On the other hand, it turns out that the same exercise with the divergence free approximation also gives the relation (A19) as the unique solution, but the vector on which the WKB approximation is done has to be defined as

$$
V \widehat{=}\left(\begin{array}{l}
X \widehat{=} r^{2} \xi_{r} \\
Y \hat{=} \delta P e^{-A}
\end{array}\right)
$$

This small difference in the definition of the second component of the vector between the divergence free ap- 


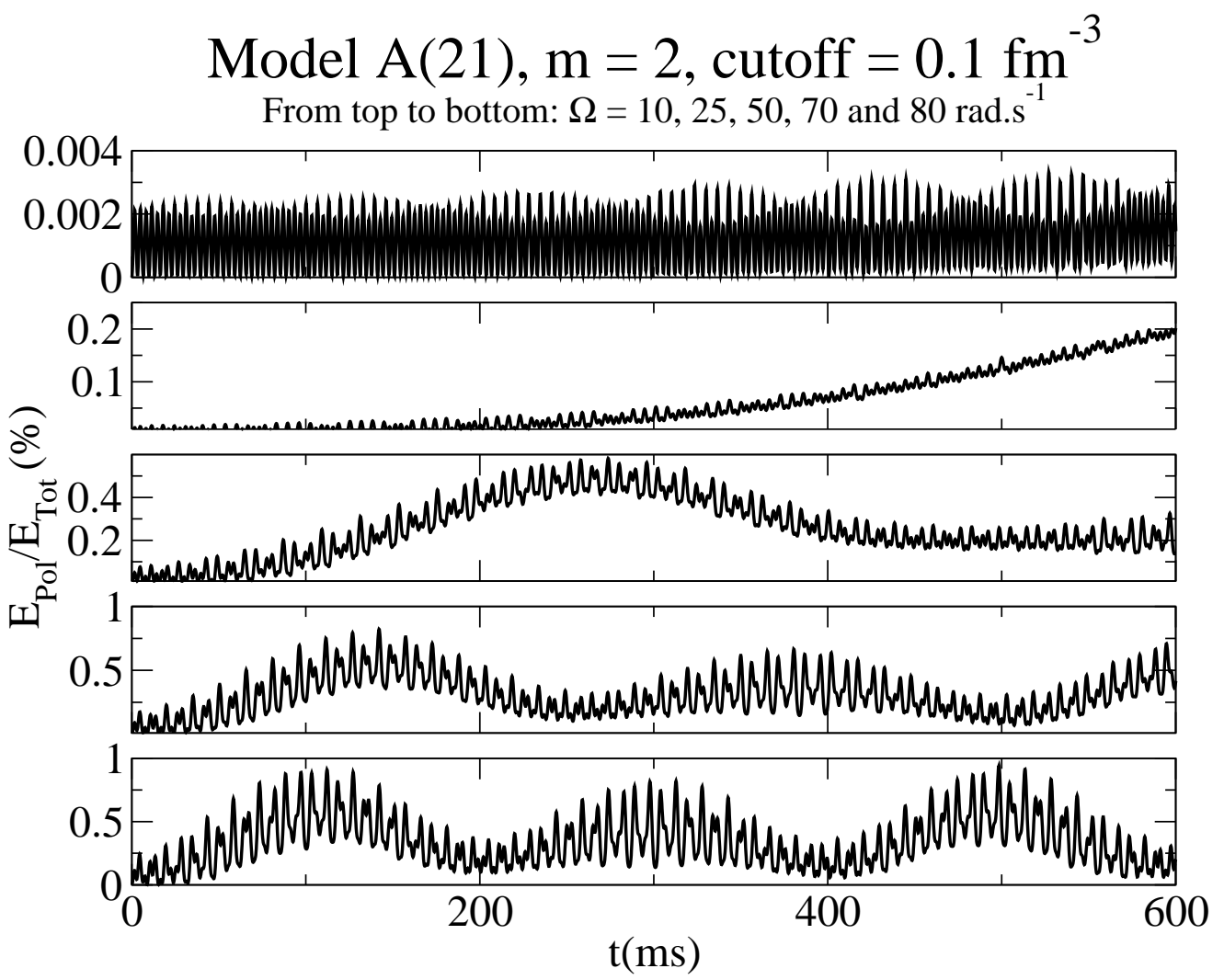

FIG. 21: Time evolution of the ratio between the polar kinetic energy and the total kinetic energy in the same nonbarotropic star as in previous figures with inertial mode for initial data.

proximation and the "exact solution" seems to indicate that the anelastic approximation (for which there is not such a difference) is closer to the exact solution than the divergence free approximation is.
[1] N. Andersson, Astrophys. J. 502, 708 (1998).

[2] J.L. Friedman and S.M. Morsink, Astrophys. J. 502, 714 (1998).

[3] J.L. Friedman and K.H. Lockitch, review in Proceedings of the 9th Marcel Grossman Meeting, edited by V. Gurzadyan, R. Jantzen and R. Ruffini, (World Scientific, Singapore), 2002.

[4] N. Andersson, Class. Quant. Grav. 20, R105 (2003).

[5] K. D. Kokkotas and J. Ruoff, review in 2001: A Relativistic Spacetime Odyssey, Proceedings of the 25th Johns Hopkins Workshop on Current Problems in Particle Theory, edited by I. Ciufolini, D. Dominici and L. Lusana (World Scientific, New Jersey), 325 (2003).

[6] S. Chandrasekhar, Phys. Rev. Lett. 24, 611 (1970).

[7] J.L. Friedman and B.F. Schutz, Astrophys. J. 221, 937 (1978); 222, 281 (1978).

[8] L. Villain, J.A. Pons, P. Cerdá-Durán and E. Gourgoulhon, Astron. \& Astrophys. 418, 283 (2004).

[9] N. Stergioulas, T.A. Apostolatos and J.A. Font, Mon. Not. of the Royal Astron. Soc. 352, 1089 (2004).
[10] L. Bildsten and G. Ushomirsky, Astrophys. J., Lett. 529, L33 (2000).

[11] L. Lindblom, B.J. Owen and G. Ushomirsky, Phys. Rev. D 62, 084030 (2000).

[12] Y. Wu, C. D. Matzner and P. Arras, Astrophys. J. 549, 1011 (2001).

[13] S. Yoshida and U. Lee, Astrophys. J. 546, 1121 (2001).

[14] J. B. Kinney and G. Mendell, Phys. Rev. D 67, 024032 (2003).

[15] S. Yoshida and U. Lee, Mon. Not. of the Royal Astron. Soc. 344, 207 (2003).

[16] S. Yoshida and U. Lee, Phys. Rev. D 67, 124019 (2003).

[17] R. Prix, G.L. Comer and N. Andersson, Mon. Not. of the Royal Astron. Soc. 348, 625 (2004).

[18] L. Villain and S. Bonazzola, Phys. Rev. D 66 , 123001 (2002).

[19] M. Prakash, T.L. Ainsworth and J.M. Lattimer, Phys. Rev. Lett. 61, 2518 (1988).

[20] D.G. Yakovlev, A.D. Kaminker, O.Y. Gnedin and P. Haensel, Phys. Rep. 354, 1 (2001). 


\title{
Model A(21), $\mathrm{m}=2$, cutoff $=0.1 \mathrm{fm}^{-3}$ (barotropic case)
}

\author{
From top to bottom: $\Omega=10,25,50,70$ and 80 rad.s $^{-1}$
}

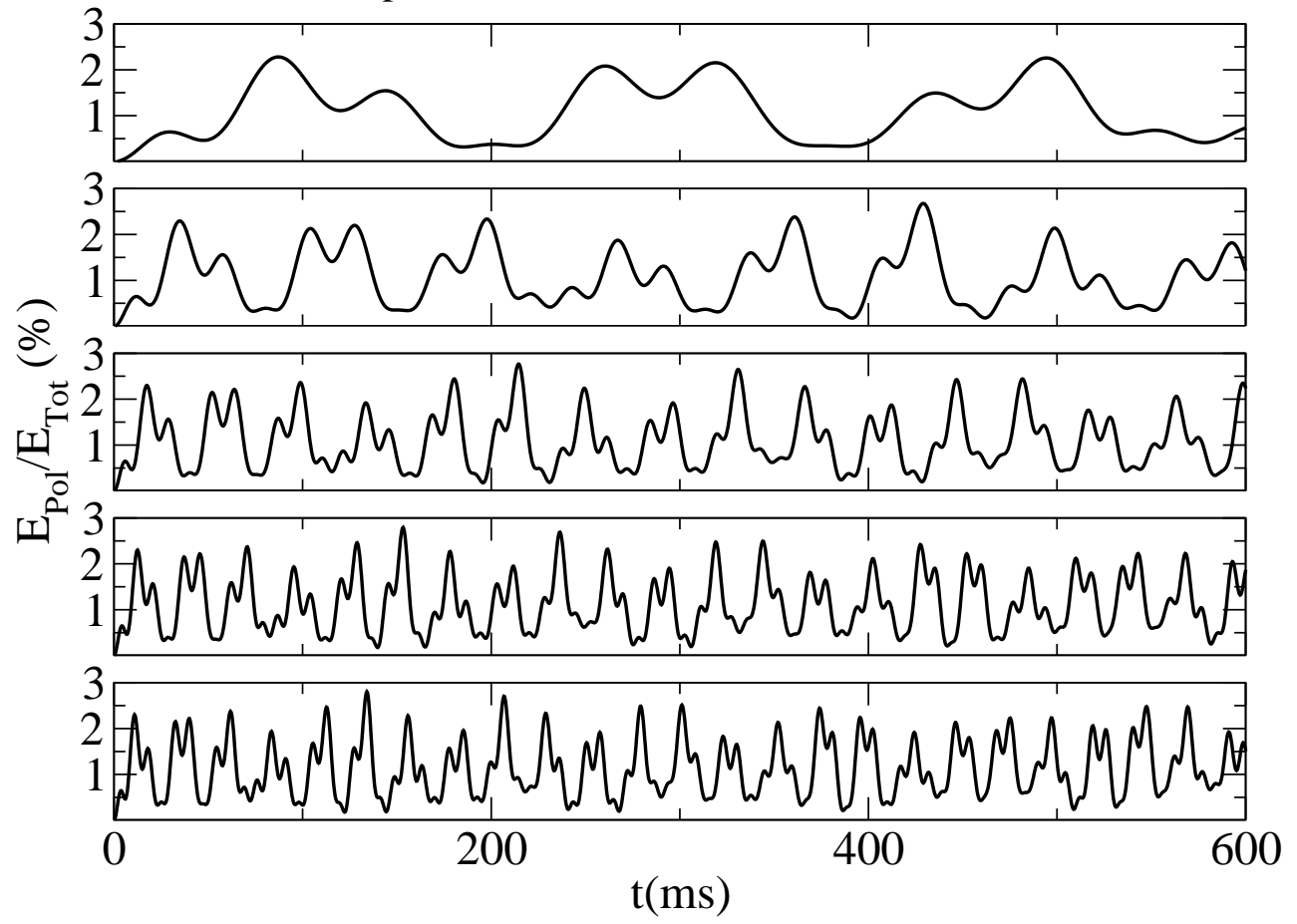

FIG. 22: Time evolution of the ratio between the polar kinetic energy and the total kinetic energy in the same barotropic star as in previous figures with inertial mode for initial data.

Dimensionless frequency of the $1=\mathrm{m}=2$ inertial mode

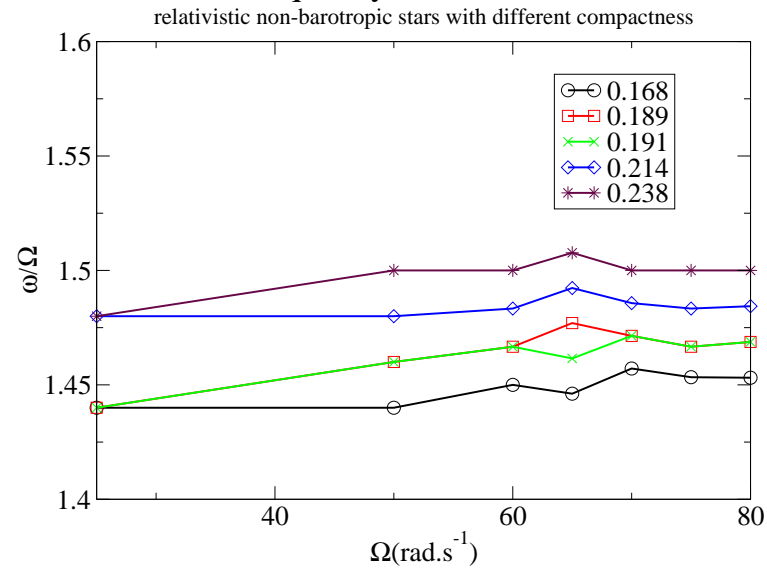

FIG. 23: Dimensionless frequency versus angular velocity of the star for $l=m=2$ inertial mode in nonbarotropic stars of various compactness. Notice that due to the fact that evolutions lasted $1 \mathrm{~s}$, the error bar is equal to $1 \mathrm{rad} . \mathrm{s}^{-1} / \Omega$. See also Fig 11 for the barotropic case.
[21] G. Baym, C. Pethick and D. Pines, Nature 224, 673 (1969).

[22] P. W. Anderson and N. Itoh, Nature 256, 25 (1975).

[23] N. Andersson, G.L. Comer and R. Prix, Phys. Rev. Lett. 90, 091101 (2003).

[24] J. Cottam, F. Paerels and M. Mendez, Nature 420, 51 (2002).

[25] R.B. Wiringa, V. Fiks and A. Fabrocini, Phys. Rev. C 38, 1010 (1988).

[26] A.Akmal, V.R. Pandharipande and D.G. Ravenhall, Phys. Rev. C 58, 1804 (1998).

[27] N. Stergioulas, Living Reviews in Relativity, 6 (2003). [Online article]: http://www.livingreviews.org/lrr-2003-3/

[28] B. Carter, Journal of Mathematical Physics 10, 70 (1969).

[29] S. Bonazzola, E. Gourgoulhon, M. Salgado and J-A. Marck, Astron. \& Astrophys. 278, 421 (1993).

[30] F. Douchin and P. Haensel, Physics Letters B 485, 107 (2000).

[31] P. Haensel, in Physics of NS interiors, edited by D. Blaschke, N.K. Glendenning and A. Sedrakian (Springer - Lecture Notes in Physics, Berlin - New York) p. 127 (2001).

[32] B. Carter, in Relativistic fluid dynamics, Lecture Notes In Mathematics 1385, edited by A. Anile and Y. 


\section{Model $\mathrm{A}(26)$ and $\mathrm{B}(24), \mathrm{m}=2$, cutoff $=0.1 \mathrm{fm}^{-3}$}

From top to bottom: $\Omega=10,25,50,70,80$ rad.s $^{-1}$

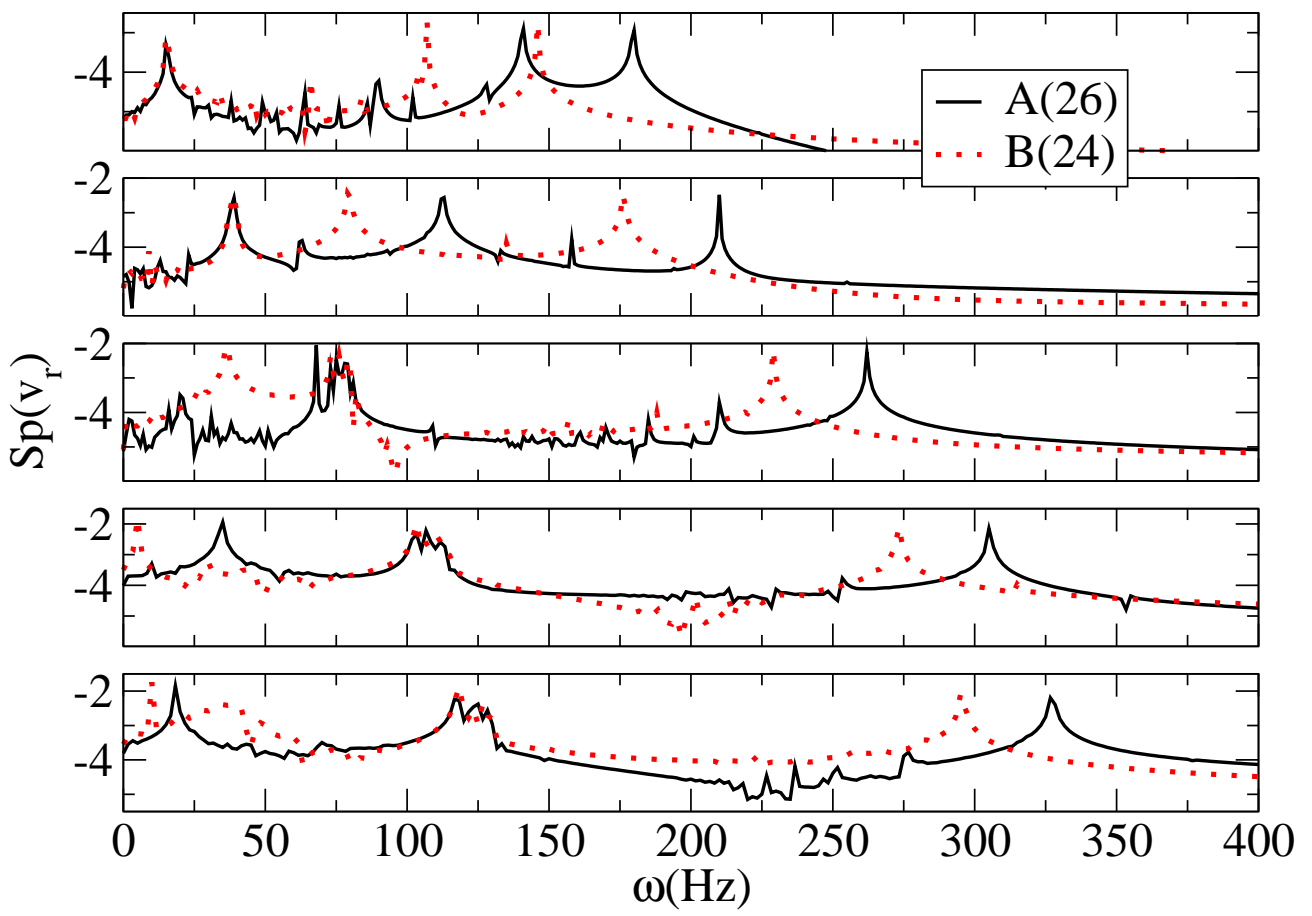

FIG. 24: Spectra of the radial velocity for several angular velocities in nonbarotropic stars of model $\mathrm{A}(26)$ and $\mathrm{B}(24)$ with inertial mode for initial data.

Choquet-Bruhat, (Springer, Berlin), 1 (1989).

[33] P. Haensel, unpublished manuscript (1980).

[34] J.M. Lattimer, C.J. Pethick, M. Prakash and P. Haensel, Phys. Rev. Lett. 66, 2701 (1991).

[35] P. Haensel, K.P. Levenfish and D.G. Yakovlev, Astron. \& Astrophys. 381, 1080 (2002).

[36] P. Haensel, K.P. Levenfish and D.G. Yakovlev, Astron. \& Astrophys. 394, 213 (2002).

[37] E. Flowers and N. Itoh, Astrophys. J. 230, 847 (1979).

[38] P.Haensel, K.P. Levenfish and D.G. Yakovlev, Astron. \& Astrophys. 372, 130 (2001)

[39] P.Haensel, K.P. Levenfish and D.G. Yakovlev, Astron. \& Astrophys. 357, 1157 (2000)

[40] C. Cutler and L. Lindblom, Astrophys. J. 314, 234 (1987).

[41] L. Zdunik, Astron. \& Astrophys. 308, 828 (1996).

[42] T.G. Cowling, Mon. Not. of the Royal Astron. Soc. 101, 367 (1941).

[43] P.N. Mc Dermott, H.M. Van Horn and J.F. Scholl, Astrophys. J. 268, 837 (1983).

[44] L. S. Finn, Mon. Not. of the Royal Astron. Soc. 232, 259 (1988).

[45] J. Novak and S. Bonazzola, J. Comp. Phys. 197/1 186 (2004).

[46] J.B. Hartle, Astrophys. J. 150, 1005 (1967).

[47] J.A. Isenberg, Waveless approximation theories of gravity, preprint University of Maryland, unpublished (1978).
[48] J.A. Isenberg and J. Nester, in General relativity and gravitation, edited by A. Held (Plenum, New York), 1, 23 (1980).

[49] J.R. Wilson and G.J. Mathews, in Frontiers in numerical relativity, edited by C.R. Evans, L.S. Finn and D.W. Hobill (Cambridge University Press, Cambridge, England), 306 (1989).

[50] G.B. Cook, S.L. Shapiro and S.A. Teukolsky, Phys. Rev. D 53, 5533 (1996).

[51] L. Blanchet, Living Reviews in Relativity, 5 (2002). [Online article]: http://www.livingreviews.org/lrr-2002-3

[52] A. Reisenegger and P. Goldreich, Astrophys. J. 395, 240 (1992).

[53] K. S. Thorne, Astrophys. J. 158, 1 (1969).

[54] L. S. Finn, Mon. Not. of the Royal Astron. Soc. 227, 265 (1987).

[55] D. Lai, Mon. Not. of the Royal Astron. Soc. 307, 1001 (1999).

[56] D. Lai, Mon. Not. of the Royal Astron. Soc. 270, 611 (1994).

[57] S. Yoshida and U. Lee, Astrophys. J. 567, 1112 (2002).

[58] G., Miniutti, J.A. Pons, E. Bert, L. Gualtieri and V. Ferrari, Mon. Not. of the Royal Astron. Soc. 338, 389 (2003).

[59] V. Ferrari, L. Gualtieri, J.A. Pons and A. Stavridis, Mon. Not. of the Royal Astron. Soc. 350, 763 (2004).

[60] U. Lee, Astron. \& Astrophys. 303, 515 (1995). 
[61] N. Andersson and G.L. Comer, Mon. Not. of the Royal Astron. Soc. 328, 1129 (2001).

[62] R. Prix and M. Rieutord, Astron. \& Astrophys. 393, 949 (2002)

[63] K.H. Lockitch, N. Andersson and J.L. Friedman, Phys. Rev. D 63, 024019 (2001).

[64] Y. Kojima, Mon. Not. of the Royal Astron. Soc. 293, 49 (1998).

[65] H.R. Beyer and K.D. Kokkotas, Mon. Not. of the Royal
Astron. Soc. 308, 745 (1999).

[66] K.H. Lockitch and N. Andersson, gr-qc/0106088 (2001).

[67] J. Ruoff, A. Stavridis and K.D. Kokkotas, Mon. Not. of the Royal Astron. Soc. 339, 1170 (2003).

[68] K.H. Lockitch, J.L. Friedman and N. Andersson, Phys. Rev. D 68, 124010 (2003).

[69] K.H. Lockitch and J.L. Friedman, Astrophys. J. 521, 764 (1999). 